\title{
Distribución horizontal de larvas de crustáceos decápodos capturadas entre Caldera e isla de Pascua (Pacífico sudoriental), octubre de 1999
}

\author{
Jorge Rivera ${ }^{1} \&$ Armando Mujica ${ }^{2}$ \\ ${ }^{1}$ Departamento de Ciencias del Mar, Universidad Arturo Prat. Casilla 121, Iquique, Chile \\ ${ }^{2}$ Facultad de Ciencias del Mar, Universidad Católica del Norte. Casilla 117, Coquimbo, Chile
}

RESUMEN. Las larvas de crustáceos decápodos son componentes importantes del meroplancton, llegando a constituir grandes concentraciones en ciertos periodos del año. No obstante, el conocimiento de la distribución y abundancia de éstas a niveles taxonómicos finos es escaso. Este trabajo analizó la distribución espacial longitudinal de las larvas de decápodos recolectadas entre Caldera y la isla de Pascua y su relación con dos variables ambientales, durante el crucero Cimar 5-islas oceánicas realizado en octubre de 1999. Se identificaron 31 unidades taxonómicas, correspondientes a grupos de decápodos pelágicos, mesopelágicos y bentónicos. Las larvas más abundantes fueron de la familia Sergestidae. La diversidad fue notoriamente variable destacándose dos máximos, uno en la zona nerítica continental y el segundo a 150 mn de la costa. Mediante análisis de ordenación (MDS) se detectó la presencia de dos comunidades de larvas de decápodos, asociadas a las condiciones de temperatura y salinidad de las aguas subantárticas relacionadas con la corriente de Humboldt y a las aguas subtropicales, respectivamente. Además, se identificaron tres ensambles entre larvas de decápodos, relacionados con el hábitat de los ejemplares adultos. Se discuten las relaciones de las larvas con su medio y con las condiciones oceanográficas y topográficas del área de estudio.

Palabras clave: larvas de crustáceos decápodos, distribución horizontal, Pacífico sudoriental, islas oceánicas chilenas.

\section{Horizontal distribution of the crustacean decapod larvae collected between Caldera and Easter Island (Southeastern Pacific), October 1999}

\begin{abstract}
The crustacean decapod larvae are a very important component of the meroplankton. They constitute high concentrations during certain seasons of the year. Nevertheless, the knowledge about their distribution and abundance at a fine taxonomic level is really poor. This paper, analyzed the longitudinal spatial distribution of the decapod larvae collected between Caldera and Easter island and the relationships with two environmental variables, during the cruise Cimar 5-oceanic islands, October 1999. The 31 taxa of larvae that correspond to three groups, pelagic, mesopelagic and benthonic decapod, were identified. Sergestid shrimp larvae were the most abundant along the track. The diversity was quite variable in the whole track, showing two picks. The first picks in the neritic area and the second to the $150 \mathrm{~nm}$ offshore. By means of an ordination analysis (MDS), the presence of two communities of decapod larvae was detected, one was associated to the conditions of temperature and salinity of the sub-antarctic waters from the Humboldt Current System and the other community to the subtropical waters, respectively. Three assemblages of decapod larvae were identified, related with the habitat of the adult individuals. The relationships of the decapod larvae with their habitat and with the oceanographic and topographic conditions in the study area are discussed.
\end{abstract}

Key words: decapod crustacean larvae, horizontal distribution, southeastern Pacific, oceanic Chilean islands. 


\section{INTRODUCCIÓN}

Los estudios sobre larvas de decápodos presentes en aguas chilenas del Pacífico sudoriental se han limitado a entregar información general de categorías taxonómicas superiores a especie (Palma, 1976, 1980; Palma et al., 1976; Mujica, 1993; Mujica \& Medina, 1997). Esto debido a que son muy escasas las descripciones de los estadios larvales en estas aguas, por lo que se hace difícil efectuar la identificación de larvas a niveles de género o especie (Wehrtmann \& Báez, 1997).

Por esta razón, el conocimiento a nivel específico de la distribución espacio-temporal de las larvas en esta área del Pacífico es escaso, a excepción de Emerita analoga Stimpson, Pleuroncodes monodon H. Milne Edwards, Petrolisthes violaceus Guérin, P. laevigatus Guérin y Allopetrolisthes angulosus Guérin (Palma, 1976, 1994; Ulloa \& Palma, 1998).

En este contexto, los estudios de distribución y abundancia de larvas de decápodos han estado restringidos a los primeros $200 \mathrm{~km}$ (100 mn) de la costa (Báez \& Martin, 1992; Palma, 1976, 1980; Ulloa \& Palma, 1998), quedando sin información la extensa zona oceánica que se proyecta desde la costa hasta la isla de Pascua.

Esta zona oceánica es influenciada por una serie de corrientes y masas de aguas, caracterizándose entre el continente y aproximadamente los $82^{\circ} \mathrm{W}$ por la presencia de aguas subantárticas entre 0 y $100 \mathrm{~m}$ de profundidad; y hacia al oeste de los $82^{\circ} \mathrm{W}$, por aguas subtropicales entre 0 y $300 \mathrm{~m}$ de profundidad, que alcanzan la región insular ubicada en la zona central del giro anticiclónico del Pacífico sur (R. Fuenzalida, com. pers.). Si bien la oceanografía costera ha sido mejor estudiada (Strub et al., 1998), las características alrededor de isla de Pascua se han descrito en estudios ocasionales de corta duración, que indican la presencia de aguas subtropicales con mayores valores de temperatura y salinidad (Silva, 1992; Olivares \& Moraga, 1993).

Desde este punto de vista, las principales masas de agua y la dirección de los flujos de corrientes, influyen en los patrones de distribución y retención de larvas y crustáceos pelágicos (McWilliam \& Phillips, 1983; Griffiths \& Brandt, 1983; Criales \& McGowan, 1994; Yeung \& McGowan, 1991), pudiendo estos patrones ser influenciados por la presencia de estuarios, pulsos de surgencia y eventos cálidos como El Niño (Báez \& Martin, 1992; Pitts, 1999; Natunewicz \& Epifanio, 2001).

El objetivo de este trabajo fue analizar la distribución horizontal de las larvas de crustáceos decápodos y describir su estructura comunitaria en la zona oceánica comprendida entre Caldera e isla de Pascua y su relación con la temperatura y salinidad registrada en octubre de 1999.

\section{MATERIALES Y MÉTODOS}

El transecto de estudio fue perpendicular a la costa sudamericana, desde Caldera $\left(27^{\circ} 00^{\prime} \mathrm{S}, 71^{\circ} 46^{\prime} \mathrm{W}\right)$ hasta isla de Pascua $\left(27^{\circ} 00^{\prime} \mathrm{S}, 107^{\circ} 35^{\prime} \mathrm{W}\right)$, en el cual se efectuaron 32 estaciones oceanográficas del 16 al 28 de octubre de 1999 (Fig.1). Las muestras de zooplancton se recolectaron con redes Bongo de 59 $\mathrm{cm}$ de diámetro y $300 \mathrm{~m}$ de abertura de malla, provistas de medidores de flujo, que se arrastraron en forma doble oblicua entre la superficie y $200 \mathrm{~m}$ de profundidad.

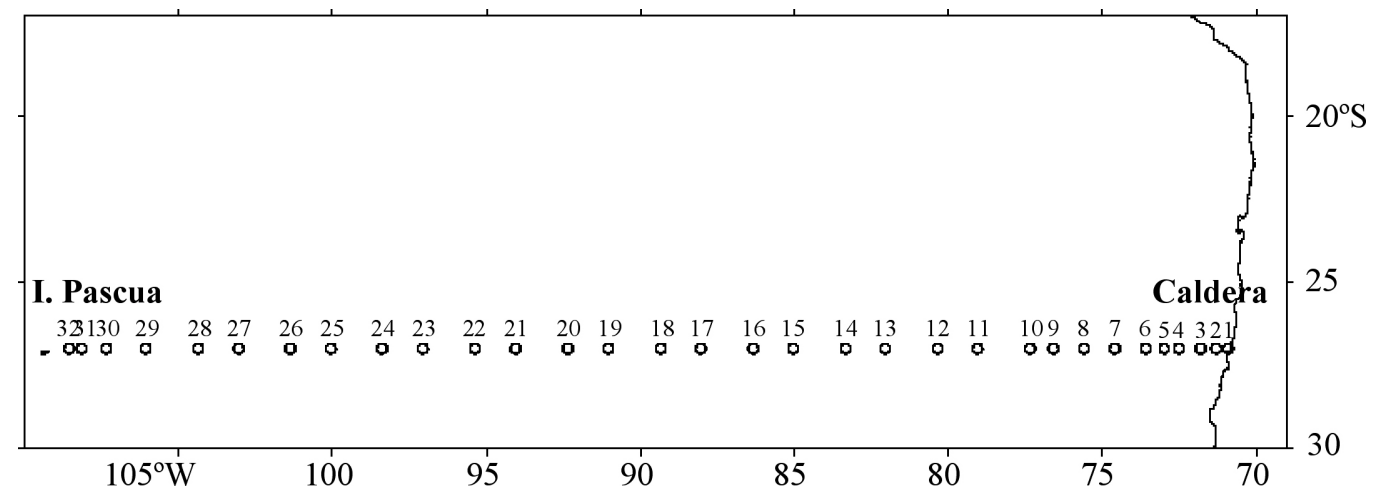

Figura 1. Ubicación de las estaciones oceanográficas en el transecto oceánico entre Caldera e isla de Pascua.

Figure 1. Location of the oceanographic stations in the oceanic transect between Caldera and Easter Island. 
Las muestras de plancton se fijaron en formalina $(5 \%)$ y se preservaron en etanol $\left(96^{\circ}\right)$. En el laboratorio se extrajo la totalidad de larvas de crustáceos decápodos y se determinó su abundancia relativa, estandarizada en número de individuos por $1000 \mathrm{~m}^{3}$ de agua filtrada (ind. $1000 \mathrm{~m}^{-3}$ ). Las larvas se identificaron al nivel taxonómico más bajo posible, mediante el uso de claves y literatura especializada (Tabla 1). También se determinó la longitud total, desde la punta del rostro hasta el borde posterior del telson, con un microscopio con micrómetro ocular graduado.

En cada estación se obtuvieron registros de temperatura $\left({ }^{\circ} \mathrm{C}\right)$ y salinidad (psu) con una sonda perfiladora CTDO-SBE25. Los muestreos se efectuaron en forma continua durante horas diurnas y nocturnas.

El análisis ecológico consistió en determinar la relación entre la abundancia y diversidad de larvas de decápodos. Luego, la abundancia y distribución de los taxa se compararon mediante un análisis de disimilitud faunística entre estaciones (tipo Q) y entre especies (tipo R), utilizando el índice de Bray Curtis y se resumieron mediante escalamiento multidimencional no métrico (MDS) (Field et al., 1982).

Para determinar la relación entre la abundancia relativa total y por taxa, y las variables abióticas, se efectuó un análisis de correlación de Pearson (Zar, 1994). Para tal efecto se calculó el promedio ponderado de temperatura y salinidad en los primeros $100 \mathrm{~m}$ de profundidad, debido a que en este estrato se encontró una capa de mezcla bastante desarrollada (R. Fuenzalida, com. pers.).

El promedio ponderado de temperatura y salinidad se calculó de acuerdo a la siguiente expresión (Rosales, 1992; Ulloa \& Palma, 1998):

$$
X_{i j}=\frac{\sum_{K=1} Z_{j k} * C_{i j k}}{\sum Z_{j k}}
$$

donde:

$X_{i j}$ : promedio ponderado del i-ésimo parámetro de la j-ésima estación

$Z_{j k}:$ k-ésima profundidad de la j-ésima estación

$C_{i j k}:$ valor del i-ésimo parámetro en la j-ésima estación a la k-ésima profundidad.

Por otra parte, a las ordenaciones realizadas en el análisis ecológico se sobrepusieron los datos de tem- peratura y salinidad, comparando en forma gráfica el efecto conjunto de las variables abióticas con los patrones de distribución observados, los que se contrastaron mediante un análisis ANOVA(Field et al., 1982; Zar, 1984).

\section{RESULTADOS}

En 31 de las 32 estaciones distribuidas entre Caldera e isla de Pascua (97\%), se identificaron larvas de crustáceos decápodos, con excepción de la estación 28 en que no se encontraron larvas. En cuanto a las posibles diferencias entre la cantidad de larvas colectadas de día y noche, se determinó que no se encontraron diferencias significativas $\left(\mathrm{p}_{\mathrm{obs}}=0,99>\mathrm{p}\right.$ $=0,05$ ), por lo que las muestras diurnas y nocturnas se trataron de la misma forma.

\section{Composición taxonómica y diversidad}

La composición taxonómica de las larvas comprendió un total de 31 unidades taxonómicas, correspondientes a 8 especies, 10 géneros, 11 familias y 2 morfos indeterminados (Tabla 1).

Los datos obtenidos mostraron la presencia de distintas fases de desarrollo en cada unidad taxonómica, destacando la ausencia de los primeros estadios larvales, como zoea I, en las familias de los decápodos braquiuros, como Pleuroncodes monodon (Galatheidae), Emerita analoga (Hippidae), y en los camarones dendrobranquiados (Sergestes spp. y Gennadas sp.), encontrándose solo larvas superiores a los estadios de nauplius y protozoea. La mayoría de las larvas colectadas correspondió a estadios con cierto grado de desarrollo, lo que se vio reflejado en los rangos de talla registrados (Tabla 1).

En general, los valores de riqueza taxonómica y diversidad específica (Shannon-Wiener) indicaron dos máximos de diversidad, el primero en las estaciones ubicadas cerca de la costa (Est. 2 y 3), con 14 unidades taxonómicas, seguido por una notable disminución de estos índices en las estaciones siguientes (Est. 4, 7 y 10). El segundo máximo se observó en la estación 11 con 6 unidades, seguido por una oscilación de la diversidad y riqueza taxonómica hasta alcanzar un nuevo incremento en las cercanías de isla de Pascua (Fig. 2).

La riqueza taxonómica, con respecto a la abundancia relativa de cada estación, mostró cierta tendencia a una relación positiva, aproximándose a una 
Tabla 1. Larvas de crustáceos decápodos identificadas en el transecto oceánico entre Caldera e isla de Pascua. Table 1. Description of crustacean decapod larvae identified in the oceanic transect between Caldera and Easter Island.

\begin{tabular}{|c|c|c|c|}
\hline $\begin{array}{l}\text { Unidad } \\
\text { taxonómica }\end{array}$ & $\begin{array}{c}\text { Estadio } \\
\text { larval }\end{array}$ & $\begin{array}{l}\text { Longitud } \\
\text { total }(\mathrm{mm})\end{array}$ & $\begin{array}{l}\text { Referencias } \\
\text { bibliográficas }\end{array}$ \\
\hline Gennadas sp. & mysis I-IV & $3,24-6,69$ & $\begin{array}{l}\text { Heldt (1938) } \\
\text { Criales \& McGowan (1993) } \\
\text { Rivera \& Guzmán (2002) }\end{array}$ \\
\hline Sergestidae (Sergestes) & $\begin{array}{l}\text { elaphocaris, } \\
\text { acanthosoma } \\
\text { y mastigopus }\end{array}$ & $0,82-6,00$ & $\begin{array}{l}\text { Knight \& Omori (1982) } \\
\text { Gurney \& Lebour (1940) } \\
\text { Calazans (1993) }\end{array}$ \\
\hline Nematocarcinus sp. & zoea avanzada & 7,08 & $\begin{array}{l}\text { Iwasaki \& Nemoto (1987) } \\
\text { Vereschaka (1990) }\end{array}$ \\
\hline Acanthephyra sp. & zoeas & $5,89-6,68$ & Báez (1997), Boschi (1981) \\
\hline Oplophorus novazeelandiae & Postlarva & 10,12 & Hendrickx \& Estrada-Navarrete (1996) \\
\hline Rhynchocinetes typus & zoeas II y III & $2,78-3,22$ & Albornoz \& Wehrtmann (1997) \\
\hline Periclimenes sp. & zoea III & 4,58 & Parker (1985) \\
\hline Synalpheus spinifrons & zoea & 2,98 & Albornoz \& Wehrtmann (1997) \\
\hline Hippolytidae sp. & zoeas avanzadas & $6,80-8,02$ & Hart (1971) \\
\hline Lysmata sp. & zoea avanzadas & $8,80-10,15$ & Parker (1985) \\
\hline Pandalidae sp. & zoeas & $5,70-7,11$ & Hart (1971) \\
\hline Heterocarpus sp. & zoea III a postlarvas & $3,74-8,02$ & Menon (1972), Felder et al. (1985) \\
\hline Crangonidae & zoeas tempranas & $2,40-2,66$ & Hart (1971), Makarov (1968) \\
\hline Neotrypaea uncinata & zoea II & 5,72 & Aste \& Retamal (1984) \\
\hline Thalassinidae morfo D.I. & zoea avanzada & 7,63 & Gurney (1938) \\
\hline Scyllarus delfíni & phyllosoma-V & 8,24 & Báez (1973), Ito \& Lucas (1990) \\
\hline Pagurus sp. & zoea II & 2,08 & Crain \& McLaughlin (1993) \\
\hline Parapagurus sp. & zoeas avanzadas & $5,22-7,01$ & Williamson \& Von Levetsow (1966) \\
\hline Pleuroncodes monodon & Zoeas II-IV & $3.10-5,02$ & Fagetti \& Campodonico (1971) \\
\hline Cevimunida johni & zoeas avanzadas & $4,02-6,80$ & Fagetti (1960), Seridji (1995) \\
\hline Galathea sp. & zoeas & $3,32-4,68$ & Gore (1979), Seridji (1995) \\
\hline Emerita analoga & zoeas II-V & $3,02-6,62$ & Johnson \& Lewis (1942) \\
\hline Leucosiidae & zoeas tempranas & $2,66-3,00$ & Boschi (1981) \\
\hline Majidae (Acanthonichinae) & zoeas I y II & $2,30-3,00$ & Boschi (1981), Hiyodo et al. (1994) \\
\hline Corystidae sp. & zoeas II-IV & $2,80-3,88$ & Boschi (1981), Paula (1996) \\
\hline Cancridae sp. & zoeas II-IV & $2,38-4,52$ & $\begin{array}{l}\text { Fagetti (1960), Boschi (1981) } \\
\text { Hong \& Ingle (1987), Báez (1997) }\end{array}$ \\
\hline Portunidae sp. & zoea & 3,82 & Boschi (1981), Báez (1997) \\
\hline Xanthidae sp. 1 & zoeas II-IV & $2,88-4,42$ & Boschi (1981), Báez (1997) \\
\hline Xanthidae sp. 2 & zoea II & $3,66-3,86$ & Boschi (1981), Báez (1997) \\
\hline Brachyura sp. 1 & megalopa sp. 1 & $4,22-6,96$ & Sin referencia \\
\hline Brachyura sp. 2 & megalopa sp .2 & $3,90-4,00$ & Sin referencia \\
\hline
\end{tabular}


asíntota (Fig. 3). La abundancia y el número de unidades taxonómicas fueron más altas en las estaciones más cercanas al continente (Est. 2 y 3), que en aquellas situadas en el transecto a isla de Pascua.

\section{Análisis de disimilitud faunística}

\section{Análisis normal (Tipo Q)}

El análisis de disimilitud faunística mostró una ordenación (MDS), que entregó una buena representación gráfica de la segregación de estaciones, con un nivel de stress de 0,12 . Se observó la presencia de dos grandes zonas en el transecto de estudio, que se diferenciaron principalmente por la composición y abundancia larval en cada una de ellas (Fig. 4).

La primera zona (zona A) comprendió las estaciones 1 a 6 , entre el continente y $150 \mathrm{mn}$ aproximadamente, caracterizándose por presentar las mayores abundancias relativas, que alcanzaron al $10,0 \%$ y $15,4 \%$ en las estaciones 2 y 3 respectivamente. En éstas se registraron las mayores densidades de larvas capturadas en el área de estudio (171 y 263 ind. $1000 \mathrm{~m}^{-3}$ ).

La segunda zona (zona B), comprendió las estaciones oceánicas situadas entre 150 y 2000 mn aproximadamente, con abundancias relativas com-

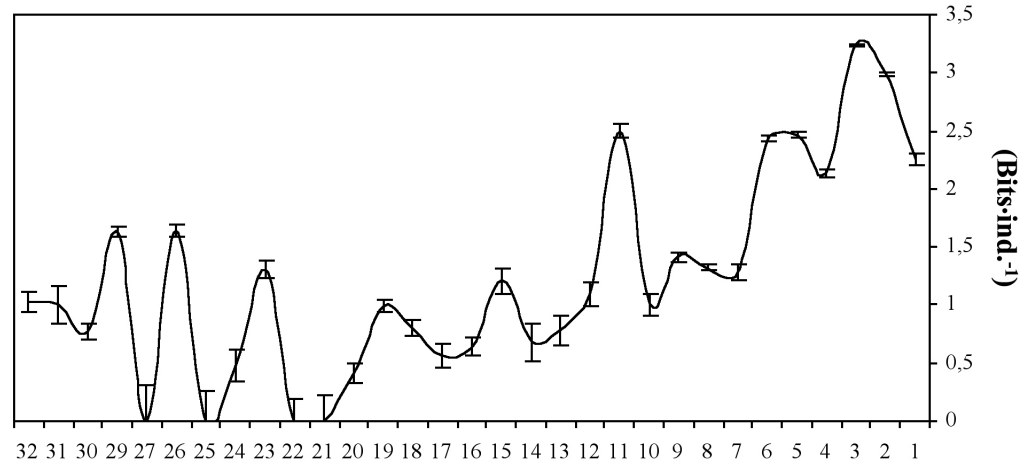

Estación

Figura 2. Índice de diversidad (Shannon-Wiener) y desviación estándar en las estaciones de muestreo.

Figure 2. Diversity index (Shannon-Wiener) and standard deviation of the sampling stations.

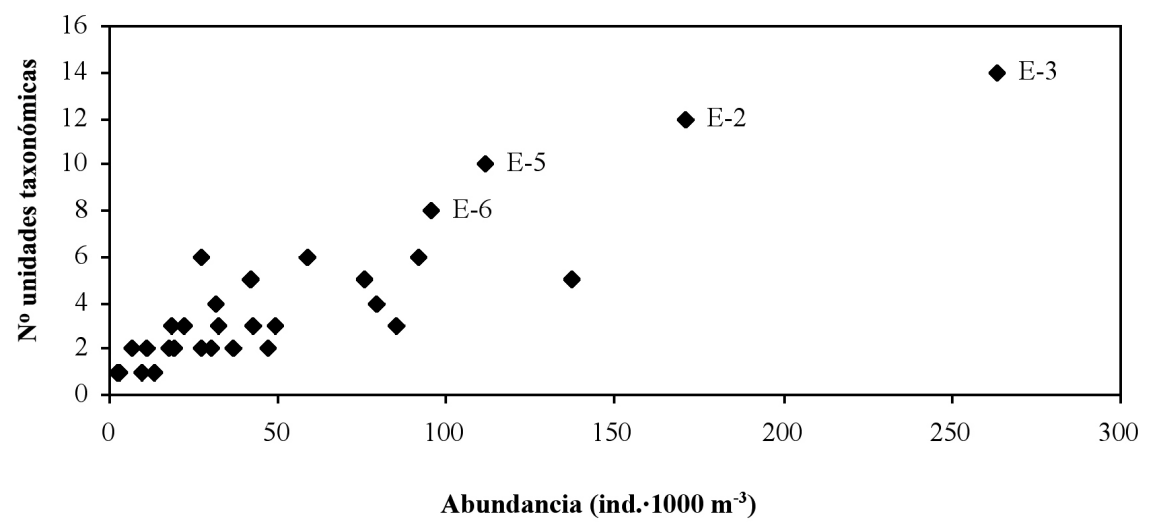

Figura 3. Diversidad versus abundancia de larvas de decápodos (ind. $1000 \mathrm{~m}^{-3}$ ) en el transecto Caldera-isla de Pascua. La diversidad está expresada como el número de unidades taxonómicas. Los números cercanos a los puntos corresponden a la estación.

Figure 3. Diversity versus abundance of the decapod larvae (ind. $1000 \mathrm{~m}^{-3}$ ) for the Caldera-Easter Island transect. Diversity is expressed as number of taxonomic unities. Numbers near points are coded by station. 
prendidas entre $0,1 \%$ y $8,0 \%$ del total de larvas de decápodos.

\section{Análisis inverso (Tipo R)}

Al comparar las disimilitudes entre las diferentes unidades taxonómicas, sobre la base de su presencia o ausencia en las estaciones, se obtuvo una ordenación (MDS) representativa de la separación de los principales conjuntos de larvas y se distinguió la presencia de dos conjuntos principales (Fig. 5).

El primer conjunto estuvo formado por larvas del género Gennadas y de la familia Sergestidae, que mostraron un fuerte grado de asociación entre ambas. Estas larvas se hallaron en las zonas A y B.

El segundo conjunto, fue conformado por el resto de las unidades taxonómicas pertenecientes a lar-

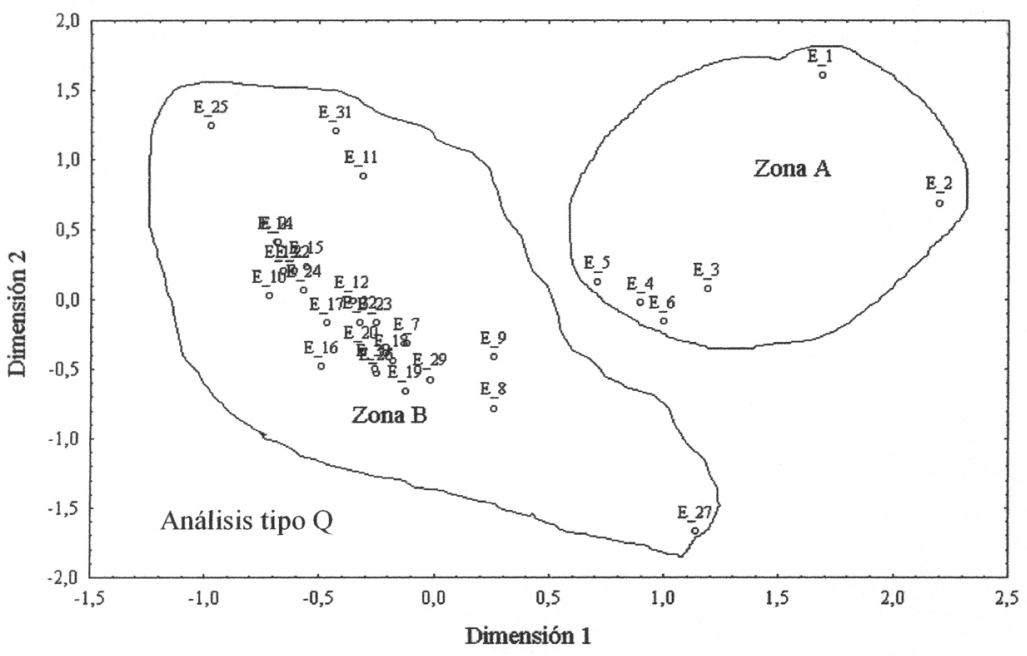

Figura 4. Ordenación (MDS) de las 31 estaciones entre Caldera e isla de Pascua, en un espacio bidimensional separando la Zona A y Zona B. Stress 0,12.

Figure 4. Ordinations (MDS) of the 31 stations between Caldera and Easter Island, in a bidimensional space separated by the Zone $A$ and Zone B. Stress 0.12 .

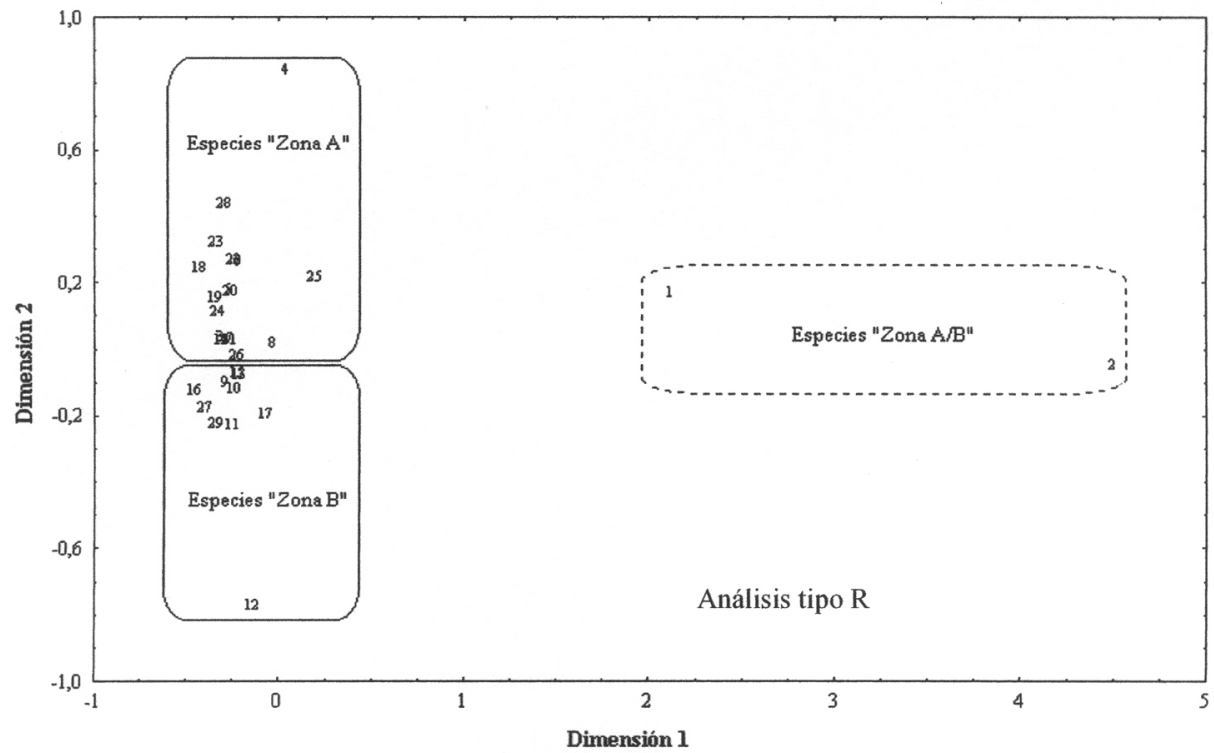

Figura 5. Ordenación (MDS) donde se observa la separación de los principales conjuntos de larvas de decápodos presentes entre Caldera e isla de Pascua. Especies Zona A/B (línea entrecortada), especies Zona A y especies Zona B los números y nombres se indican en la Tabla 3. Stress $0,07$.

Figure 5. Inverse ordinations (MDS) showing separated the principal assemblages of decapod larvae present between Caldera and Easter Island. Zone A/B species (dashed line), Zone A species and Zone B species, the numbers and names are listed in Table 3. Stress 0.07. 
vas de decápodos bentónicos y de aguas profundas. Sin embargo, a un nivel menor de disimilitud, se distinguió la presencia de dos subconjuntos dentro de este gran grupo de larvas. Uno que agrupó los diferentes taxa de decápodos (braquiuros, anomuros y carideos) asociados con las aguas más cercanas a la plataforma continental (zona A) y el otro que reunió al resto de larvas de decápodos que están principalmente asociadas a los montes submarinos localizados en el trayecto hacia isla de Pascua (zona B).

\section{Distribución horizontal y abundancia relativa}

La distribución y las clases de abundancias equidistantes según la escala métrica $\log ^{2}$, para el total de larvas de decápodos, estuvo marcada por los mayores valores en las primeras estaciones del transecto, alcanzando valores máximos de 263 ind. $1000 \mathrm{~m}^{-3}$ en la estación 3. Se observó una marcada disminución de abundancia de este a oeste, con valores mínimos de 79 y 2 ind. $1000 \mathrm{~m}^{-3}$ en las estaciones 19 y 25, respectivamente. Esta tendencia se mantuvo relativamente constante hasta la isla de Pascua (Fig. 6a). En los grupos mayores del orden Decapoda, se observaron diferentes patrones de distribución y abundancia, que se detallan en la Tabla 2.

\section{Dendrobranchiata (Fig. 6b)}

Este grupo que consideró las familias Aristeidae (Gennadas) y Sergestidae, presentó las mayores dominancias numéricas alcanzando al $11,1 \%$ y $40,4 \%$ respectivamente. Las mayores abundancias se encontraron en las estaciones 6 y 8 (27 y 94 ind. $1000 \mathrm{~m}^{-3}$ ), ubicadas entre 100 y $250 \mathrm{mn}$ de la costa.

De acuerdo a los grupos formados en el análisis de disimilitud faunística entre las estaciones, se determinó que en la zona $\mathrm{A}$, las larvas de los dendrobranchiados fueron relativamente escasas, registrándose éstas a partir de la estación 3. Las mayores densidades se encontraron entre el límite de la zona A y la zona B.

En la zona B, las larvas de Gennadas sp. fueron poco constantes, desapareciendo en tramos amplios del transecto y reapareciendo cerca de isla de Pascua. Las larvas de Sergestidae spp. se mantuvieron constantes en todas las estaciones de la zona B, con una fuerte dominancia numérica con respecto a los otros grupos, aunque inferior a 63 ind. $1000 \mathrm{~m}^{-3}$.

\section{Caridea (Fig. 6c)}

Este infraorden, que agrupó el mayor número de unidades taxonómicas identificadas a nivel de familia, se distribuyó a lo largo de casi todo el transecto, presentando las mayores abundancias en la zona A (55 ind. $\left.1000 \mathrm{~m}^{-3}\right)$.

En Caridea, la distribución y abundancia relativa de cada familia presentó patrones diferenciados entre ellas. La familia Pandalidae estuvo representada por dos unidades taxonómicas a lo largo del transecto, considerándose como un grupo accesorio con la mayor densidad de larvas dentro de Caridea (114 ind. $1000 \mathrm{~m}^{-3}$ ). No obstante, esta abundancia fue producto del alto número de larvas de Heterocarpus, que estuvo presente sólo en la zona A, con valores de 43 ind. $1000 \mathrm{~m}^{-3}$ (Est. 2), pero sin alcanzar las aguas oceánicas (zona B). Por el contrario, las larvas de Pandalidae sp. se registraron sólo en la zona $\mathrm{B}$, y con una baja abundancia, que no superó los 12 ind. $1000 \mathrm{~m}^{-3}$ (Est. 10).

La familia Oplophoridae, representada por dos unidades taxonómicas (Acanthephyra sp. y Oplophorus novaezeelandiae), presentó una amplia distribución, la cual estuvo constituida principalmente por las larvas de Acanthephyra, que estuvieron en ambas zonas (A y B), aunque la mayor densidad se registró en la zona $\mathrm{B}$ ( 8 ind. $1000 \mathrm{~m}^{-3}$ ). Las larvas de $O$. novaezeelandiae solo se registraron en la estación 11, a $440 \mathrm{mn}$ de la costa y con escasa abundancia (Tabla 2).

La familia Hippolytidae agrupó una forma larval indeterminada (Hippolytidae sp.) y a las larvas del género Lysmata. Ambos taxa presentaron bajas abundancias, inferiores a 5 ind. $1000 \mathrm{~m}^{-3}$. Las larvas de Hippolytidae sp. se encontraron en la zona A y las del género Lysmata en las estaciones cercanas a la isla de Pascua (zona B).

El resto de las familias de Caridea fueron Rhynchocinetidae, Alpheidae, Crangonidae, Nematocarcinidae y Palaemonidae, las cuales se distribuyeron en forma diferente en ambas zonas. En la zona A se colectaron larvas de Rhynchocinetes typus (Rhynchocinetidae) y Synalpheus spinifrons (Alpheidae), en escasa abundancia, inferior a 10 ind. $1000 \mathrm{~m}^{-3}$.

Las tres familias restantes también se encontraron en baja densidad, (<6 ind. $1000 \mathrm{~m}^{-3}$ ) y estuvieron restringidas sólo a la zona $B$. Las larvas de 

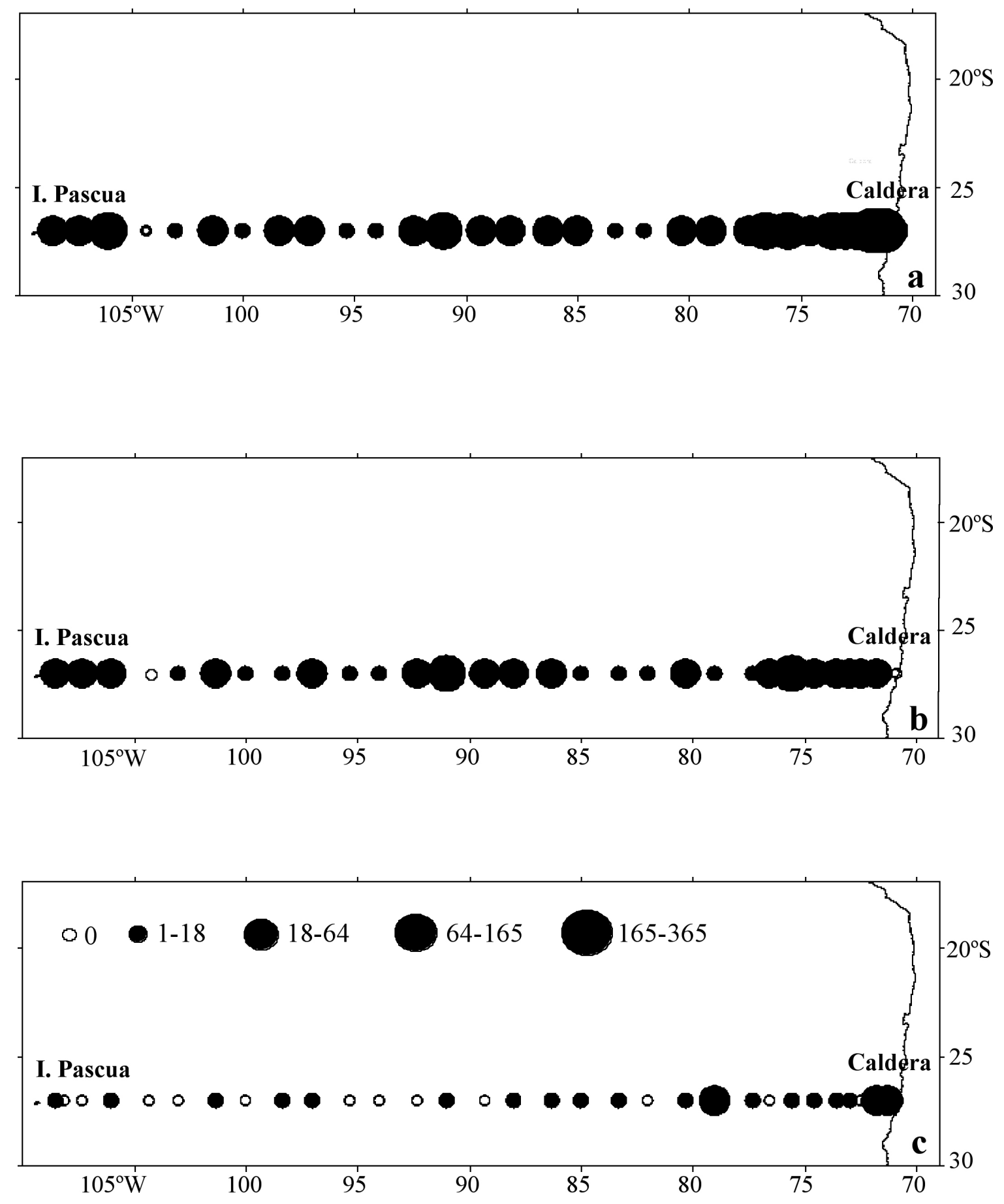

Figura 6. Distribución horizontal de larvas (ind. $1000 \mathrm{~m}^{-3}$ ) entre Caldera e isla de Pascua. a) Crustáceos decápodos, b) Dendrobranchiata, c) Caridea, d) Macrura, e) Anomura y f) Brachyura. 

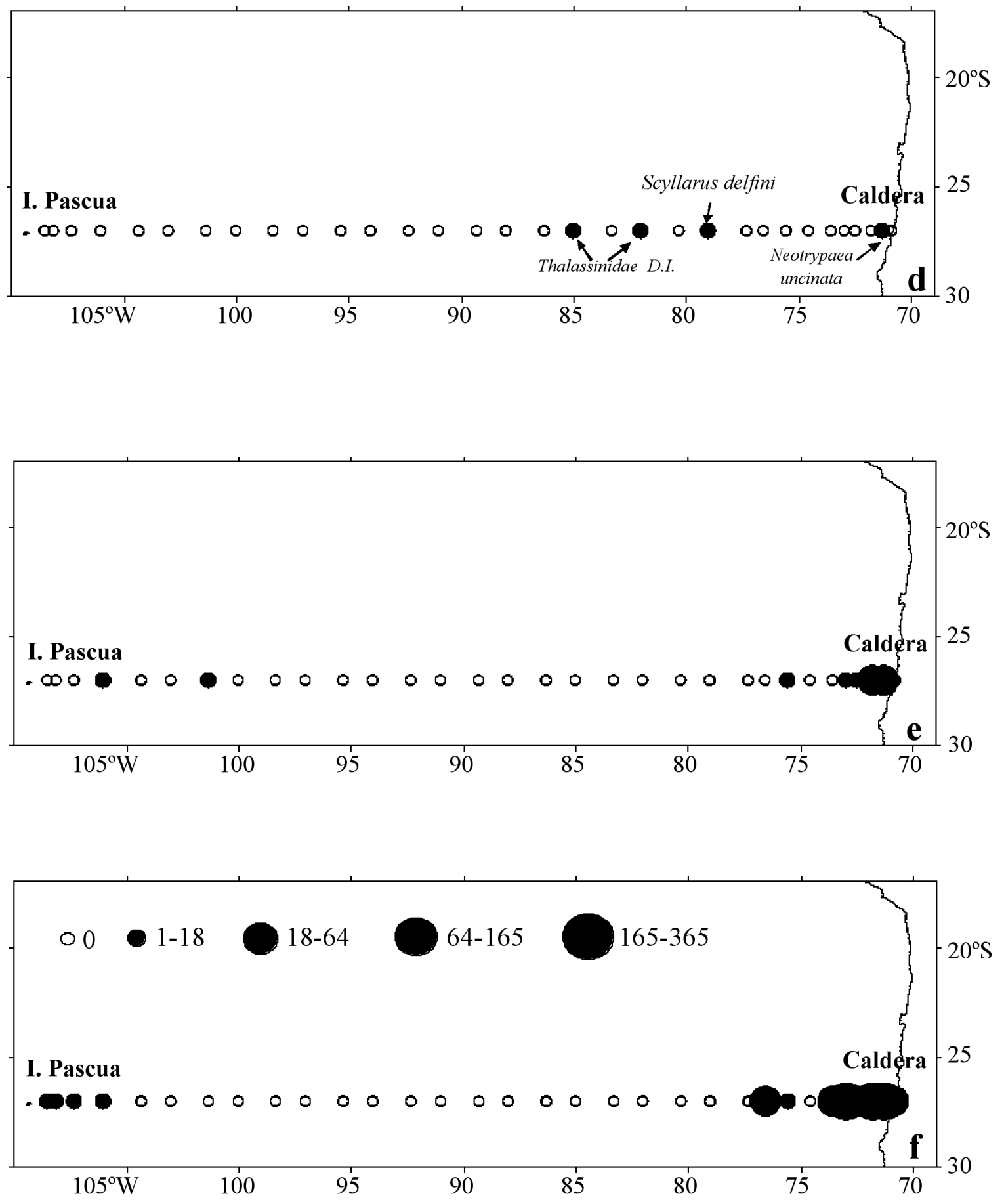

Figures 6. Horizontal distribution of larvae (ind. $\cdot 1000 \mathrm{~m}^{-3}$ ) between Caldera and Easter Island. a) Decapod crustacean b) Dendrobranchiata, c) Caridea, d) Macrura, e) Anomura and f) Brachyura. 
Tabla 2. Abundancia (ind. $1000 \mathrm{~m}^{-3}$ ) de larvas de crustáceos decápodos registradas en el transecto oceánico entre Caldera e Isla de Pascua en octubre de 1999.

Table 2. Abundance (ind. $1000 \mathrm{~m}^{-3}$ ) of crustacean decapod larvae recorded in the oceanic transect between Caldera and Easter Island during October 1999.

\begin{tabular}{|c|c|c|c|c|c|c|c|c|c|c|c|c|c|c|c|c|c|c|c|c|c|c|c|c|c|c|c|c|c|c|c|c|}
\hline \multicolumn{33}{|c|}{ Estaciones } \\
\hline Unidad Taxonómica & 1 & 2 & 3 & 4 & 5 & 6 & 7 & 8 & & 101 & 1112 & 1213 & 31 & 1415 & & 16 & 17 & 18 & 19 & 20 & 21 & 22 & 23 & 242 & 2526 & 2627 & 729 & 930 & 03 & 313 & 32 & total \\
\hline \multicolumn{33}{|l|}{ DENDROBRANCHIATA } \\
\hline Gennadas sp. & 0 & 0 & 16 & 17 & 32 & 27 & 112 & 24 & 11 & 0 & 03 & 30 & 0 & 00 & 0 & 0 & 0 & 12 & 10 & 3 & 0 & 0 & 8 & 0 & $0 \varepsilon$ & 83 & 323 & 36 & 6 & 0 & 2 & 189 \\
\hline Sergestidae spp. & 0 & 0 & 19 & 81 & 16 & 7 & 279 & 943 & 33 & 15 & 617 & 1713 & 3 & 913 & 34 & 44 & 26 & 35 & 63 & 33 & 9 & 13 & 20 & 17 & 240 & 400 & 040 & 041 & 1 & 32 & 25 & 690 \\
\hline \multicolumn{33}{|l|}{ CARIDEA } \\
\hline Synalpheus spinifrons & 0 & 4 & 0 & 0 & 0 & 0 & 0 & 0 & 0 & 0 & 0 & 0 & 0 & 0 & 0 & 0 & 0 & 0 & 0 & 0 & 0 & 0 & 0 & 0 & 0 & 00 & 0 & 0 & 0 & 0 & 0 & 4 \\
\hline Rhynchocinetes typus & 0 & 4 & 3 & 01 & 10 & 0 & 0 & 0 & 0 & 0 & 0 & 0 & 0 & 0 & 0 & 0 & 0 & 0 & 0 & 0 & 0 & 0 & 0 & 0 & 0 & 00 & 0 & 0 & 0 & 0 & 0 & 17 \\
\hline Pandalidae sp. & 0 & 0 & 0 & 0 & 0 & 0 & 0 & 0 & & 12 & 6 & 0 & 0 & 2 & 0 & 3 & 0 & 0 & 0 & 0 & 0 & 0 & 4 & 2 & 0 & 30 & 0 & 0 & 0 & 0 & 0 & 31 \\
\hline Heterocarpus sp. & 0 & 43 & 33 & 0 & 0 & 2 & 5 & 0 & 0 & 0 & 0 & 0 & 0 & 0 & 0 & 0 & 0 & 0 & 0 & 0 & 0 & 0 & 0 & 0 & 0 & 00 & 0 & 0 & 0 & 0 & 0 & 83 \\
\hline Crangonidae sp. & 0 & 0 & 0 & 0 & 0 & 0 & 0 & 0 & 0 & 0 & 6 & 0 & 0 & 0 & 0 & 0 & 0 & 0 & 2 & 0 & 0 & 0 & 0 & 0 & 0 & 00 & 0 & 0 & 0 & 0 & 0 & 8 \\
\hline Nematocarsinus sp. & 0 & 0 & 0 & 0 & 0 & 0 & 0 & 0 & 0 & 0 & 0 & 0 & 0 & 0 & 0 & 3 & 0 & 0 & 0 & 0 & 0 & 0 & 0 & 0 & 0 & 00 & 0 & 0 & 0 & 0 & 0 & 3 \\
\hline Periclimenes sp. & 0 & 0 & 0 & 0 & 0 & 0 & 0 & 0 & 0 & 0 & 0 & 0 & 0 & 0 & 0 & 0 & 0 & 0 & 0 & 0 & 0 & 0 & 0 & 0 & 0 & 30 & 0 & 0 & 0 & 0 & 0 & 3 \\
\hline Lysmata sp. & 0 & 0 & 0 & 0 & 0 & 0 & 0 & 0 & 0 & 0 & 0 & 0 & 0 & 0 & 0 & 0 & 0 & 0 & 0 & 0 & 0 & 0 & 0 & 0 & 0 & 30 & 0 & 0 & 0 & 0 & 2 & 5 \\
\hline Hippolytidae sp. & 0 & 0 & 5 & 0 & 0 & 0 & 0 & 0 & 0 & 0 & 0 & 0 & 0 & 0 & 0 & 0 & 0 & 0 & 0 & 0 & 0 & 0 & 0 & 0 & 0 & 00 & 0 & 0 & 0 & 0 & 0 & 5 \\
\hline O. novazeelandiae & 0 & 0 & 0 & 0 & 0 & 0 & 0 & 0 & 0 & 0 & 3 & 0 & 0 & 0 & 0 & 0 & 0 & 0 & 0 & 0 & 0 & 0 & 0 & 0 & 0 & 0 & 0 & 0 & 0 & 0 & 0 & 3 \\
\hline Acanthephyra sp. & 0 & 4 & 0 & 0 & 7 & 0 & 0 & 2 & 0 & 0 & 35 & 30 & 0 & 03 & 3 & 0 & 4 & 0 & 4 & 0 & 0 & 0 & 0 & 0 & 0 & 00 & 0 & 8 & 0 & 0 & 0 & 38 \\
\hline \multicolumn{33}{|l|}{ MACRURA } \\
\hline Neotrypaea uncinata & 0 & 4 & 0 & 0 & 0 & 0 & 0 & 0 & 0 & 0 & 0 & 0 & 0 & 0 & 0 & 0 & 0 & 0 & 0 & 0 & 0 & 0 & 0 & 0 & 0 & 0 & 0 & 0 & 0 & 0 & 0 & 4 \\
\hline Thalassinidae morfo D. & 0 & 0 & 0 & 0 & 0 & 0 & 0 & 0 & 0 & 0 & 0 & 0 & 4 & 0 & 3 & 0 & 0 & 0 & 0 & 0 & 0 & 0 & 0 & 0 & 0 & 0 & 0 & 0 & 0 & 0 & 0 & 7 \\
\hline Syllarus delfini & 0 & 0 & 0 & 0 & 0 & 0 & 0 & 0 & 0 & 0 & 3 & 0 & 0 & 0 & 0 & 0 & 0 & 0 & 0 & 0 & 0 & 0 & 0 & 0 & 0 & 00 & 0 & 0 & 0 & 0 & 0 & 3 \\
\hline \multicolumn{33}{|l|}{ ANOMURA } \\
\hline Pagurus sp. & 7 & 0 & 0 & 0 & 0 & 0 & 0 & 0 & 0 & 0 & 0 & 0 & 0 & 0 & 0 & 0 & 0 & 0 & 0 & 0 & 0 & 0 & 0 & 0 & 0 & 0 & 0 & 0 & 0 & 0 & 0 & 7 \\
\hline Parapagurus sp. & 0 & 9 & 5 & 0 & 0 & 0 & 0 & 2 & 0 & 0 & 0 & 0 & 0 & 0 & 0 & 0 & 0 & 0 & 0 & 0 & 0 & 0 & 0 & 0 & 0 & 00 & 0 & 0 & 0 & 0 & 0 & 16 \\
\hline Emerita analoga & 7 & 9 & 16 & 4 & 0 & 0 & 0 & 0 & 0 & 0 & 0 & 0 & 0 & 0 & 0 & 0 & 0 & 0 & 0 & 0 & 0 & 0 & 0 & 0 & 0 & 00 & 0 & 0 & 0 & 0 & 0 & 36 \\
\hline Pleuroncodes monodon & 0 & 26 & 33 & 01 & 10 & 0 & 0 & 0 & 0 & 0 & 0 & 0 & 0 & 0 & 0 & 0 & 0 & 0 & 0 & 0 & 0 & 0 & 0 & 0 & 0 & 0 & 0 & 0 & 0 & 0 & 0 & 68 \\
\hline Cervimunida johni & 0 & 0 & 8 & 0 & 0 & 0 & 0 & 0 & 0 & 0 & 0 & 0 & 0 & 0 & 0 & 0 & 0 & 0 & 0 & 0 & 0 & 0 & 0 & 0 & 0 & 0 & 0 & 0 & 0 & 0 & 0 & 8 \\
\hline Galathea sp. & 0 & 0 & 0 & 2 & 0 & 0 & 0 & 0 & 0 & 0 & 0 & 0 & 0 & 0 & 0 & 0 & 0 & 0 & 0 & 0 & 0 & 0 & 0 & 0 & 03 & 30 & 0 & 2 & 0 & 0 & 0 & 7 \\
\hline \multicolumn{33}{|l|}{ BRACHYURA } \\
\hline Leucosiidae sp. & 14 & 0 & 0 & 0 & 0 & 0 & 0 & 0 & 0 & 0 & 0 & 0 & 0 & 0 & 0 & 0 & 0 & 0 & 0 & 0 & 0 & 0 & 0 & 0 & 0 & 0 & 0 & 0 & 0 & 0 & 0 & 14 \\
\hline Majidae sp. & 0 & 4 & 8 & 0 & 0 & 0 & 0 & 0 & 0 & 0 & 0 & 0 & 0 & 0 & 0 & 0 & 0 & 0 & 0 & 0 & 0 & 0 & 0 & 0 & 0 & 0 & 0 & 0 & 0 & 0 & 0 & 12 \\
\hline Corystidae sp. & 0 & 43 & 3 & 0 & 3 & 9 & 0 & 0 & 0 & 0 & 0 & 0 & 0 & 0 & 0 & 0 & 0 & 0 & 0 & 0 & 0 & 0 & 0 & 0 & 0 & 0 & 0 & 0 & 0 & 0 & 0 & 58 \\
\hline Cancridae sp. & 0 & 0 & 41 & 275 & 523 & 34 & 01 & 14 & 41 & 0 & 0 & 0 & 0 & 0 & 0 & 0 & 0 & 0 & 0 & 0 & 0 & 0 & 0 & 0 & 0 & 0 & 0 & 0 & 0 & 0 & 0 & 210 \\
\hline Portunidae sp. & 0 & 0 & 0 & 0 & 0 & 2 & 0 & 0 & 0 & 0 & 0 & 0 & 0 & 0 & 0 & 0 & 0 & 0 & 0 & 0 & 0 & 0 & 0 & 0 & 0 & 0 & 0 & 0 & 0 & 0 & 0 & 2 \\
\hline Xanthidae sp.1 & 7 & 13 & 14 & 0 & 3 & 9 & 0 & 0 & 0 & 0 & 0 & 0 & 0 & 0 & 0 & 0 & 0 & 0 & 0 & 0 & 0 & 0 & 0 & 0 & 0 & 0 & 0 & 0 & 0 & 0 & 0 & 46 \\
\hline Xanthidae sp.2 & 0 & 0 & 0 & 0 & 0 & 0 & 0 & 0 & 0 & 0 & 0 & 0 & 0 & 0 & 0 & 0 & 0 & 0 & 0 & 0 & 0 & 0 & 0 & 0 & 0 & 0 & 0 & 2 & 2 & 0 & 0 & 4 \\
\hline Megalopasp.1 & 7 & 9 & 60 & 33 & 7 & 5 & 0 & 0 & 0 & 0 & 0 & 0 & 0 & 0 & 0 & 0 & 0 & 0 & 0 & 0 & 0 & 0 & 0 & 0 & 0 & 0 & 0 & 0 & 0 & 0 & 0 & 120 \\
\hline Megalopa sp.2 & 0 & 0 & 0 & 0 & 0 & 0 & 0 & 0 & 0 & 0 & 0 & 0 & 0 & 0 & 0 & 0 & 0 & 0 & 0 & 0 & 0 & 0 & 0 & 0 & 0 & 0 & 0 & 0 & 0 & 3 & 2 & 6 \\
\hline Total por estación & 421 & 171 & 263 & 9211 & 111 & 96 & 4313 & 37 & 852 & 272 & 2722 & 2218 & 81 & 1118 & 84 & 49 & 30 & 47 & 79 & 36 & 9 & 13 & 32 & 19 & 259 & 59 & 376 & 649 & 9 & 73 & 32 & 1707 \\
\hline Porcentaje por estación & 2 & 10 & 15 & 5 & 7 & 6 & 3 & 8 & 5 & 2 & 2 & 11 & 1 & 1 & 1 & 3 & 2 & 3 & 5 & 2 & 1 & 1 & 2 & 1 & 0 & 30 & 0 & 4 & 3 & 0 & 2 & 100 \\
\hline
\end{tabular}

Crangonidae se colectaron en las estaciones 11 y 19 (440 y $1100 \mathrm{mn}$ ); las de Nematocarcinus sp. (Nematocarcinidae) en la estación $16(800 \mathrm{mn})$ y las de Periclimenes sp. (Palemonidae) en la estación 26 (1600 mn), cercana a isla de Pascua.

\section{Macrura (Fig. 6d)}

El grupo Macrura, que no corresponde a la sistemática actualizada por Martin \& Davis (2001), pero para efectos del presente estudio se ha considerado como tal, dado que agrupó a los estadios larvales de los infraórdenes Thalassinidea y Palinura, que presentaron bajos aportes de abundancia en la zona de estudio. En este grupo, la familia Callianassidae representada por Neotrypaea uncinata, se recolectó sólo en la estación 2 (zona A).

La familia Thalassinidae representada por el morfo D.I, se encontró en la estación 13 y 15 (600 y $760 \mathrm{mn}$ ) en la zona B y la familia Scyllaridae representada por larvas de Scyllarus delfini, se encontró en la estación 11 (440 mn) de la zona B.

\section{Anomura (Fig. 6e)}

Este infraorden estuvo compuesto por seis unidades taxonómicas que se encontraron en la zona $\mathrm{A}$. En esta zona, la abundancia relativa de este grupo 
fue baja, alcanzando 16 y 33 ind. $1000 \mathrm{~m}^{-3}$ para larvas de Emerita analoga (Hippidae) y de Pleuroncodes monodon (Galatheidae) respectivamente, entre las estaciones 1 y 4.

Las larvas de Pagurus (Paguridae) y Parapagurus (Parapaguridae), también se distribuyeron solo en la zona A, entre la estación 1 y 3 , con densidades inferiores a 9 ind. $1000 \mathrm{~m}^{-3}$.

Las únicas larvas de este grupo que se encontraron en ambas zonas (A y B), pertenecieron al género Galathea (Galatheidae), registrándose una mayor ocurrencia en la zona B, en las estaciones 26 y 29 (1600 y $1800 \mathrm{mn}$ ), en las cercanías de isla de Pascua.

\section{Brachyura (Fig. 6f)}

Este infraorden se distribuyó de manera opuesta entre ambas zonas, observando una total ausencia de larvas entre las estaciones 10 y 27 (300-1700 mn), con una dominancia del $28 \%$, siendo el segundo grupo de importancia numérica a lo largo del transecto.

En este grupo, solo la familia Leucossidae se registró en la estación 1. El resto de las familias de braquiuros, alcanzó una distribución relativamente amplia, ocurriendo en al menos dos estaciones en la zona A.

Las larvas de la familia Cancridae, con una sola forma larval indeterminada, se encontraron desde la estación 3 hasta 9 (30-280 mn), con densidad de 52 ind. $1000 \mathrm{~m}^{-3}$, dominando numéricamente en la zona A.

Similar situación se observó en las larvas morfotipo indeterminado de la familia Corystidae y de megalopa sp. 1, que se distribuyeron entre la estación 2 y 6 (20-100 mn), con abundancias relativas de 43 y 60 ind. $1000 \mathrm{~m}^{-3}$ en las estaciones 2 y 3 , respectivamente.

Las larvas de la familia Xanthidae sp. 1 y la subfamilia Acanthonychinae de la familia Majidae, se distribuyeron entre el continente y las primeras $30 \mathrm{mn}$, aunque en escasa densidad (<14 ind. 1000 $\left.\mathrm{m}^{-3}\right)$.

Tres unidades taxonómicas de Brachyura se encontraron alejadas del continente, como la familia Portunidae, registrada en la estación 6 y las larvas de la familia Xanthidae sp. 2 y de megalopa sp. 2 , que se encontraron en el extremo opuesto del transecto, más cercanas a isla de Pascua.

\section{Condiciones oceanográficas}

Las condiciones oceanográficas entre Caldera e isla de Pascua fueron analizadas por Fuenzalida (com. pers.). Los promedios ponderados de temperatura y salinidad (0-100 m), reflejaron un incremento gradual desde el continente hacia isla de Pascua, observando las mayores variaciones verticales térmicas y salinas en las primeras 9 estaciones (Figs. 7a y 7b). Las restantes estaciones, de características más oceánicas, mantuvieron prácticamente invariables el perfil vertical, tanto en temperatura como en salinidad, sugiriendo una mayor estabilidad en esta zona.

La temperatura en las estaciones 1 a 6 presentó valores entre 12,06 y $13,26^{\circ} \mathrm{C}$, relacionada con aguas de origen subantártico de la corriente de Humboldt. Las estaciones 7 a 9 presentaron valores entre 14,05 y $15,15^{\circ} \mathrm{C}$, mientras que el resto de las estaciones, desde la 10 hasta la 32, registraron temperaturas superiores a $17^{\circ} \mathrm{C}$, asociadas a aguas cálidas subtropicales (Fig. 7a).

Las estaciones 1 a 9 conformaron el grupo de baja salinidad asociado a aguas frías de la corriente de Humboldt, con valores entre 34,34 y 34,67 psu, mientras que el resto de las estaciones relacionadas con aguas subtropicales, registraron valores que se incrementaron desde 34,69 psu en la estación 10 , hasta 36,12 psu en la estación 32 (Fig. 7b).

\section{Relación entre los datos biológicos y oceanográficos}

La relación entre la abundancia relativa de las larvas de decápodos y las dos variables ambientales por estación, según el análisis de correlación de Pearson, proporcionó correlaciones significativas de tipo inverso (Tabla 4). Esto significó que al aumentar los valores promedios ponderados de temperatura y salinidad (0-100 m), disminuyó la abundancia relativa de larvas.

Por otra parte, al sobreponer los valores de temperatura y salinidad en cada estación, en las zonas A y B, formadas según la distribución de la abundancia de las larvas de decápodos (Fig. 4), se observó gráficamente que los grupos denominados zona "A" con respecto a la zona "B", se separaron por las características de temperatura y salinidad (Figs. 8a y 8b), existiendo diferencias significativas entre ambas zonas (ANOVA, $\mathrm{p}<0,05$ ), indicando que estas dos variables estarían relacionadas con la distribución y abundancia relativa de las larvas de 


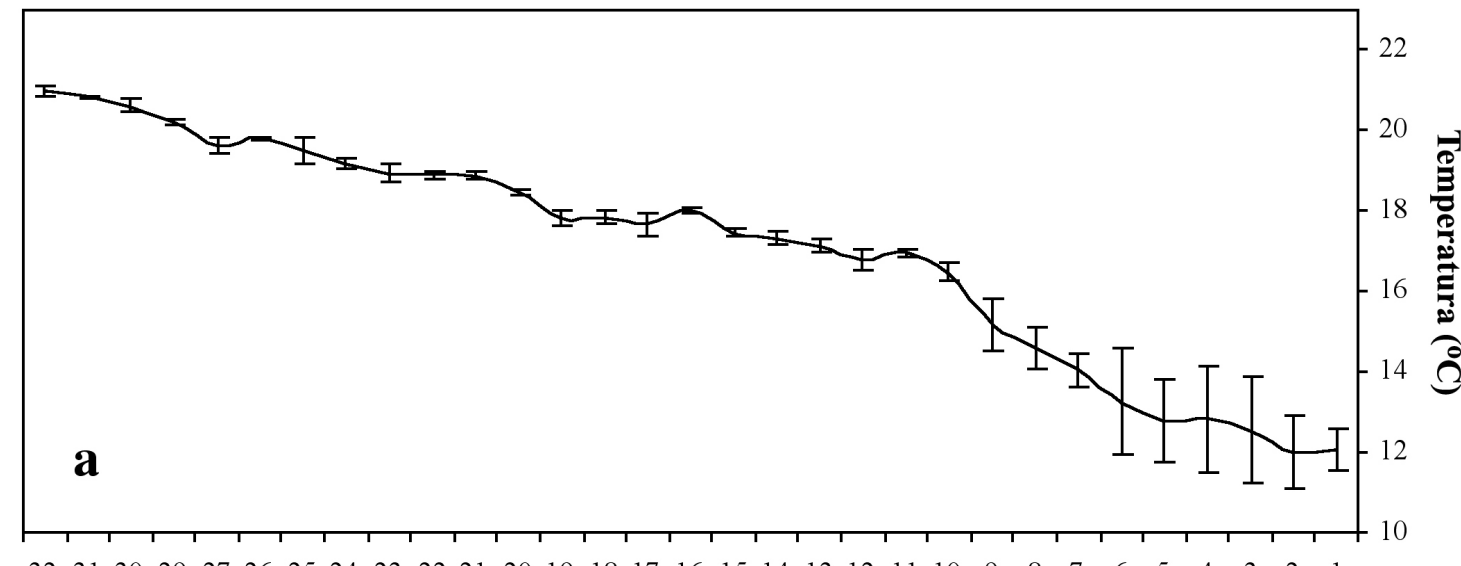

$\begin{array}{llllllllllllllllllllllllllllllll}32 & 31 & 30 & 29 & 27 & 26 & 25 & 24 & 23 & 22 & 21 & 20 & 19 & 18 & 17 & 16 & 15 & 14 & 13 & 12 & 11 & 10 & 9 & 8 & 7 & 6 & 5 & 4 & 3 & 2 & 1\end{array}$

Estación

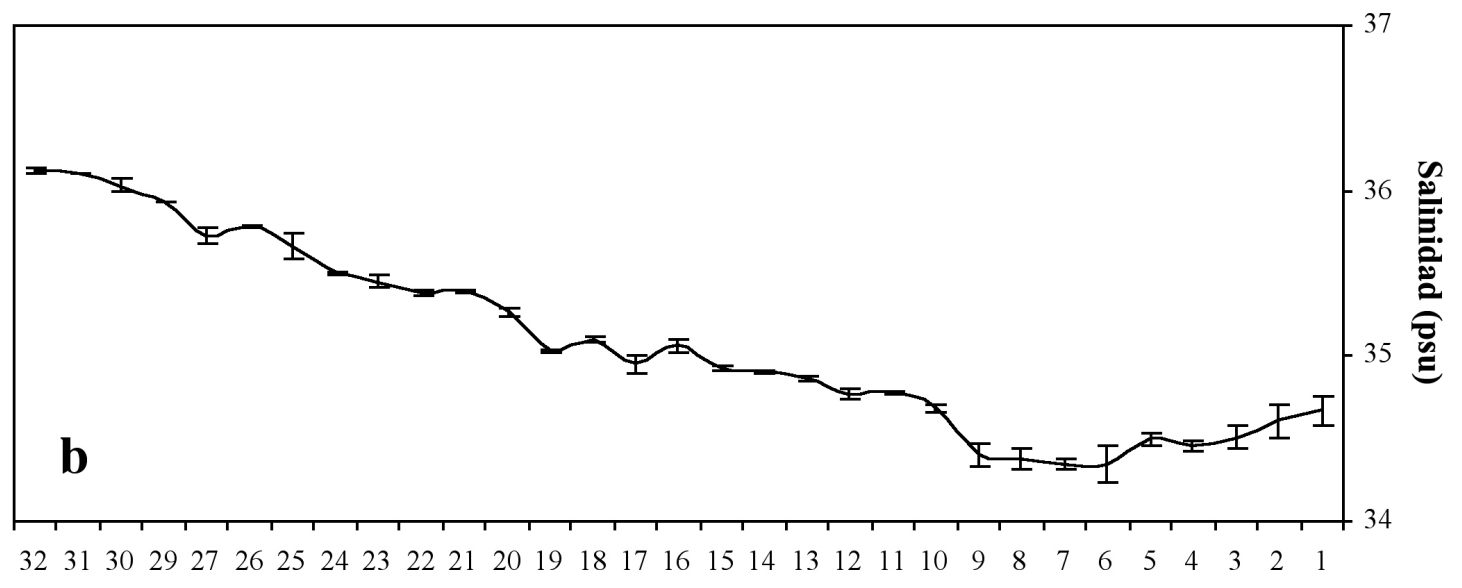

\section{Estación}

Figura 7. Promedios ponderados verticales $(0-100 \mathrm{~m})$ y rango de variabilidad de: a) temperatura $\left({ }^{\circ} \mathrm{C}\right)$ y b) salinidad (psu), en las estaciones del transecto Caldera-isla de Pascua.

Figure 7. Vertical weighted means $(100 \mathrm{~m})$ and variability range of: a) temperature $\left({ }^{\circ} \mathrm{C}\right)$ and b) salinity (psu), in each stations of the transect Caldera-Easter Island.

decápodos en el transecto de Caldera a isla de Pascua.

Las correlaciones obtenidas entre las siete unidades taxonómicas con mayores porcentajes de dominancia (Gennadas sp., Sergestidae spp., Pandalidae, Galtheidae, Corystidae sp., Cancridae sp., Xanthidae sp. 1), reflejaron diferentes grados de relación con las variables ambientales (Tabla 5).

La mayoría de los taxa se relacionaron significativamente con la temperatura, observándose los valores más altos en las larvas de las familias
Cancridae sp. y Xanthidae sp.1, con un $\mathrm{r}^{2}$ de 0,4 y 0,5 respectivamente. Estas relaciones fueron mucho menores con la salinidad, obteniéndose mayores valores en estas mismas familias (Tabla 5).

Las larvas Cancridae sp., Xanthidae sp. 1, Corystidae sp., Heterocarpus sp. y Pleuroncodes monodon, presentaron una distribución similar, separándose ampliamente de Sergestidae y Gennadas sp., encontrándose asociadas a salinidades entre 34,43 y $34,53 \mathrm{psu}$, y temperaturas entre 12,43 y $13,42^{\circ} \mathrm{C}$. 
Tabla 3. Distribución de las unidades taxonómicas colectadas entre Caldera e isla de Pascua. Table 3. Distribution of the taxonomic unities collected between Caldera and Easter Island.

\begin{tabular}{|c|c|c|c|c|}
\hline $\mathbf{N}^{\circ}$ & Unidades taxonómicas & Zona A & Zona B & Zonas A y B \\
\hline & DENDROBRANCHIATA & & & \\
\hline 1 & Gennadas sp. & & & $X$ \\
\hline \multirow[t]{2}{*}{2} & Sergestidae spp. & & & $\mathrm{X}$ \\
\hline & CARIDEA & & & \\
\hline 3 & Synalpheus spinifrons & $X$ & & \\
\hline 5 & Rhynchocinetes typus & $\mathrm{X}$ & & \\
\hline 12 & Pandalidae sp. & & $\mathrm{X}$ & \\
\hline 6 & Heterocarpus sp. & $X$ & & \\
\hline 8 & Crangonidae sp. & & $\mathrm{X}$ & \\
\hline 9 & Nematocarcinus sp. & & $\mathrm{X}$ & \\
\hline 10 & Periclimenes sp. & & $\mathrm{X}$ & \\
\hline 11 & Lysmata sp. & & $X$ & \\
\hline 7 & Hippolytidae sp. & $\mathrm{X}$ & & \\
\hline 13 & Oplophorus novazeelandiae & & $\mathrm{X}$ & \\
\hline \multirow[t]{2}{*}{4} & Acanthephyra sp. & $X$ & & \\
\hline & MACRURA & & & \\
\hline 14 & Neotrypaea uncinata & $\mathrm{X}$ & & \\
\hline 16 & Thalassinidae morfo D.I & & $\mathrm{X}$ & \\
\hline \multirow{2}{*}{15} & Syllarus delfini & & $\mathrm{X}$ & \\
\hline & ANOMURA & & & \\
\hline 17 & Pagurus sp. & $X$ & & \\
\hline 20 & Parapagurus sp. & $\mathrm{X}$ & & \\
\hline 19 & Emerita analoga & $\mathrm{X}$ & & \\
\hline 21 & Pleuroncodes monodon & $\mathrm{X}$ & & \\
\hline 22 & Cervimunida johni & $X$ & & \\
\hline \multirow[t]{2}{*}{18} & Galathea sp. & & $X$ & \\
\hline & BRACHYURA & & & \\
\hline 26 & Leucosiidae sp. & $X$ & & \\
\hline 25 & Majidae sp. & $\mathrm{X}$ & & \\
\hline 23 & Corystidae sp. & $\mathrm{X}$ & & \\
\hline 27 & Cancridae sp. & $\mathrm{X}$ & & \\
\hline 28 & Portunidae sp. & & $\mathrm{X}$ & \\
\hline 24 & Xanthidae sp. 1 & $X$ & & \\
\hline 29 & Xanthidae sp. 2 & & $X$ & \\
\hline 30 & Megalopa sp. 1 & $X$ & & \\
\hline 31 & Megalopa sp. 2 & & $\mathrm{X}$ & \\
\hline
\end{tabular}


Para analizar las características ambientales donde se distribuyeron las larvas más abundantes, se graficaron los rangos y el valor promedio ponderado de temperatura y salinidad entre todas las estaciones del transecto $\left(17,06^{\circ} \mathrm{C}\right.$ y 35,09 psu) (Fig. 9). Se determinó que las larvas de la familia Sergestidae y del género Gennadas (Aristeidae), presentaron una distribución media similar, asociándose a salinidades promedio de 35,07 psu y temperaturas medias de $16,85-17,33^{\circ} \mathrm{C}$, producto del amplio rango de distribución que presentaron ambos taxa. Esta situación fue contraria para el resto de los grupos taxonómicos contemplados en este análisis, observando una distribución asociada a bajos valores promedios de temperatura y salinidad, debido a su distribución más costera (Fig. 9).

Tabla 4. Valores de correlación entre la abundancia de larvas de decápodos y las variables ambientales. El asterisco indica los valores significativos $(\mathbf{p}<0,01)$.

Table 4. Correlations values between the abundance of decapod larvae and the environmental variables The asterisk indicate the significant values $(\mathbf{p}<0.01)$.

\begin{tabular}{|lccc|}
\hline Variables & $\begin{array}{c}\text { Coeficiente } \\
\text { Pearson } \\
(\mathbf{r})\end{array}$ & $\begin{array}{c}\text { Coeficiente de } \\
\text { determinación } \\
\left(\mathbf{r}^{2}\right)\end{array}$ & $\mathbf{p}$ \\
\hline Temperatura & $-0,65$ & 0,42 & $0,000083^{*}$ \\
Salinidad & $-0,47$ & 0,22 & $0,007244^{*}$ \\
\hline
\end{tabular}

\section{DISCUSIÓN}

\section{Diversidad y estructura comunitaria}

Los mayores valores de riqueza taxonómica y de diversidad (H') se determinaron en las estaciones más cercanas al continente, frente a Caldera, seguidos de una marcada disminución hacia las estaciones oceánicas. Esto concuerda con lo reportado por Báez \& Martin (1992) para la zona norte de Chile $\left(18^{\circ}\right.$ y $\left.28^{\circ} \mathrm{S}\right)$ y por Palma $(1976,1980)$ frente a Valparaíso, quienes determinaron una alta diversidad de larvas en aguas costeras. Sin embargo, estos trabajos sólo consideraron estaciones oceánicas hasta $100 \mathrm{mn}$ de la costa.

En el presente trabajo se observó una considerable disminución de la diversidad taxonómica entre las estaciones 7 y 10 , siendo esta una tendencia general de las larvas de crustáceos al alejarse de la costa (Sastry, 1983), que se relacionaría con la dispersión y la ontogenia propia de estos organismos (McConaugha, 1992; Anger, 2001).

Por otra parte, la presencia de un segundo máximo de diversidad en la estación 11 (Fig. 2), puede estar relacionado con la cercanía de las islas Desventuradas y de montes submarinos en ese sector (Vereschaka, 1990; Parin et al., 1997). O bien, por encontrarse estas estaciones sobre una zona de transición o de borde entre dos sistemas biológicos, como se describe a la unión de dos comunidades o ecotono (McGowan, 1972; Brower et al., 1997). Esto último, puede ocurrir entre el Sistema de la Corriente de Humboldt ( $\mathrm{SCH}$ ) y las aguas subtropicales (Silva, 1985; Arana, 1987; Moraga \& Olivares, 1996).

Tabla 5. Valores de correlación entre los grupos taxonómicos más abundantes con las variables ambientales. En negrita se indican las correlaciones significativas $(\mathbf{p}<\mathbf{0 , 0 5})$.

Table 5. Correlations values between the most abundant taxonomic groups and the environmental variables. In bold are indicate the significant values $(\mathbf{p}<0.05)$.

\begin{tabular}{|l|ccc|ccc|}
\hline & \multicolumn{3}{|c|}{ Temperatura } & \multicolumn{3}{c|}{ Salinidad } \\
Taxa & $\mathbf{r}$ & $\mathbf{r}^{2}$ & $\mathbf{p}$ & $\mathbf{r}$ & $\mathbf{r}^{\mathbf{2}}$ & $\mathbf{p}$ \\
\hline Gennadas sp. & $-0,217$ & 0,047 & 0,242 & $-0,178$ & 0,032 & 0,337 \\
Sergestidae spp. & 0,232 & 0,054 & 0,210 & 0,006 & 0,000 & 0,977 \\
Pandalidae & $-0,396$ & 0,156 & 0,028 & $-0,322$ & 0,104 & 0,077 \\
Galatheidae & $-0,405$ & 0,164 & 0,024 & $-0,165$ & 0,027 & 0,376 \\
Corystidae sp. & $-0,573$ & 0,329 & 0,001 & $-0,364$ & 0,132 & 0,044 \\
Cancridae sp. & $-0,634$ & 0,402 & 0,000 & $-0,571$ & 0,326 & 0,001 \\
Xanthidae sp. 1 & $-0,712$ & 0,506 & 0,000 & $-0,433$ & 0,187 & 0,015 \\
\hline
\end{tabular}


Esta situación fue detectada mediante el análisis ecológico tipo Q, permitiendo observar la separación de dos comunidades de larvas de decápodos dentro del transecto analizado. No obstante, esta separación ocurrió entre las estaciones 6 y 7, alrededor de las $150 \mathrm{mn}$ de la costa (Fig. 4).

Si bien, el límite físico entre ambos sistemas no está definido con certeza aún (R. Fuenzalida, com. pers.), ambas zonas ecológicas serían equivalentes a las definidas por las condiciones oceanográficas del océano Pacífico sudoriental (Silva \& Sievers, 1974; Sievers \& Silva, 1975; Silva \& Konow, 1975; Silva, 1992; Moraga \& Olivares, 1996). La zona A correspondería al $\mathrm{SCH}$ relacionada con aguas subantárticas, y por otro lado, la zona B se relacionaría con aguas subtropicales correspondientes al giro anticiclónico del Pacífico sur. Al respecto, se observó un cambio apreciable en la composición taxonómica y abundancia relativa de larvas de decápodos, siendo esta última fuertemente relacionada con las variables ambientales consideradas (Figs. 8a y 8b).

En este sentido, la elevada abundancia de larvas dentro de la zona del SCH (zona A) correspondiente a un sistema altamente productivo, y la notable disminución de larvas en la zona de aguas subtropicales (zona B), característico de un sistema oligotrófico que presenta bajos niveles de biomasa zooplanctónica y valores relativamente altos de diversidad (McGowan, 1971; Parson et al., 1984; Mann \& Lazier, 1991), son patrones ecológicos que sólo se pueden observar a meso y macroescala, entre espacios de cientos a miles de kilómetros (Haury et al., 1978; Mann \& Lazier, 1991).

El aumento en la diversidad de larvas dentro de aguas subtropicales (zona B), se observó en las cercanías de las islas Desventuradas (Est. 11), registrándose un phyllosoma de Scyllarus delfini
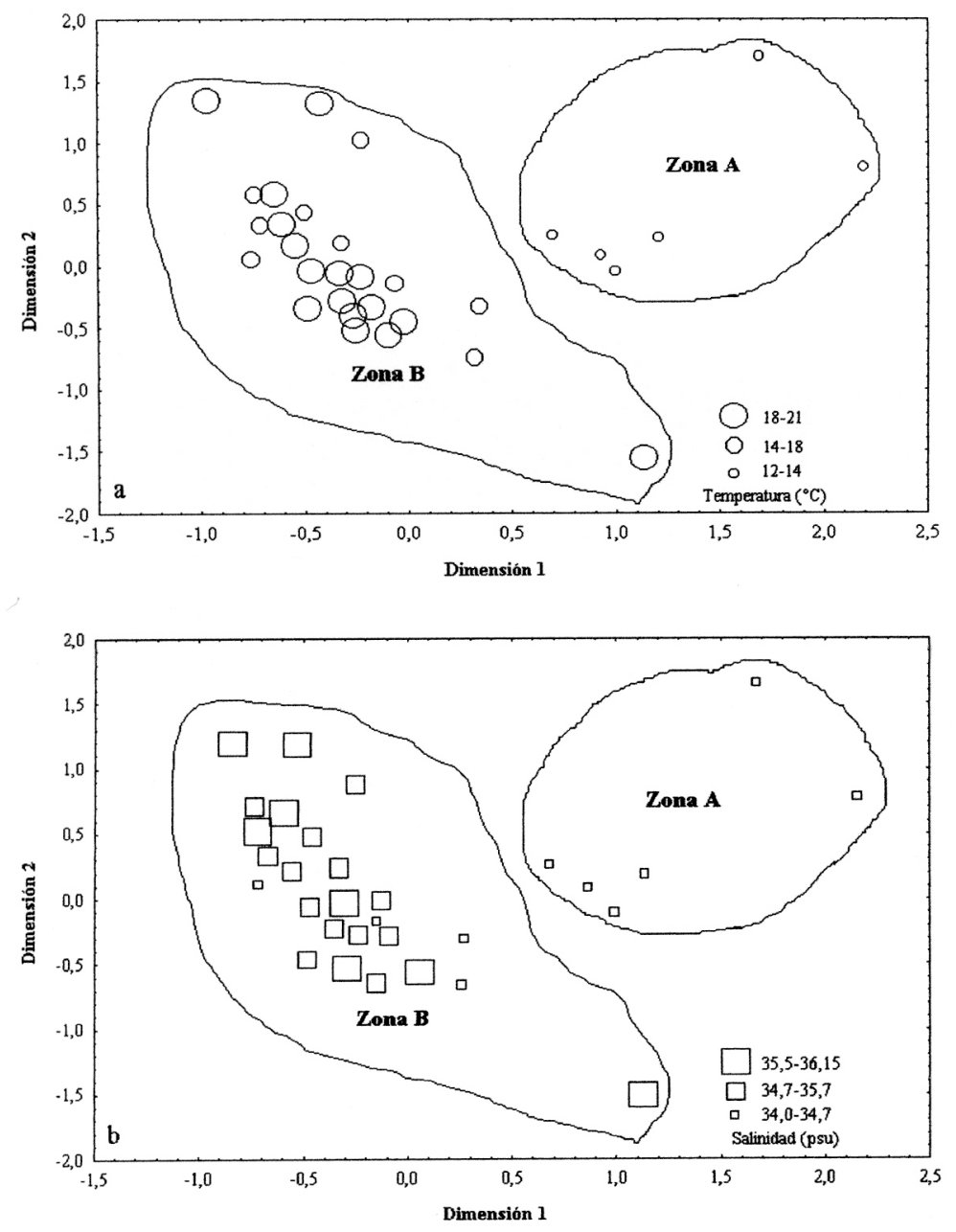

Figura 8. Relación de los grupos de estaciones delineados en la Figura 6 con las variables ambientales según la sobreposición de los valores. a) relación con la temperatura $\left({ }^{\circ} \mathbf{C}\right)$ y b) relación con la salinidad (psu).

Figure 8. Relationship of the groups of stations delineated in the Figure 6 with the environmental variables based on the superimposed of the values. a) relationship with the temperature $\left({ }^{\circ} \mathrm{C}\right)$ and b) relationship with the salinity (psu). 


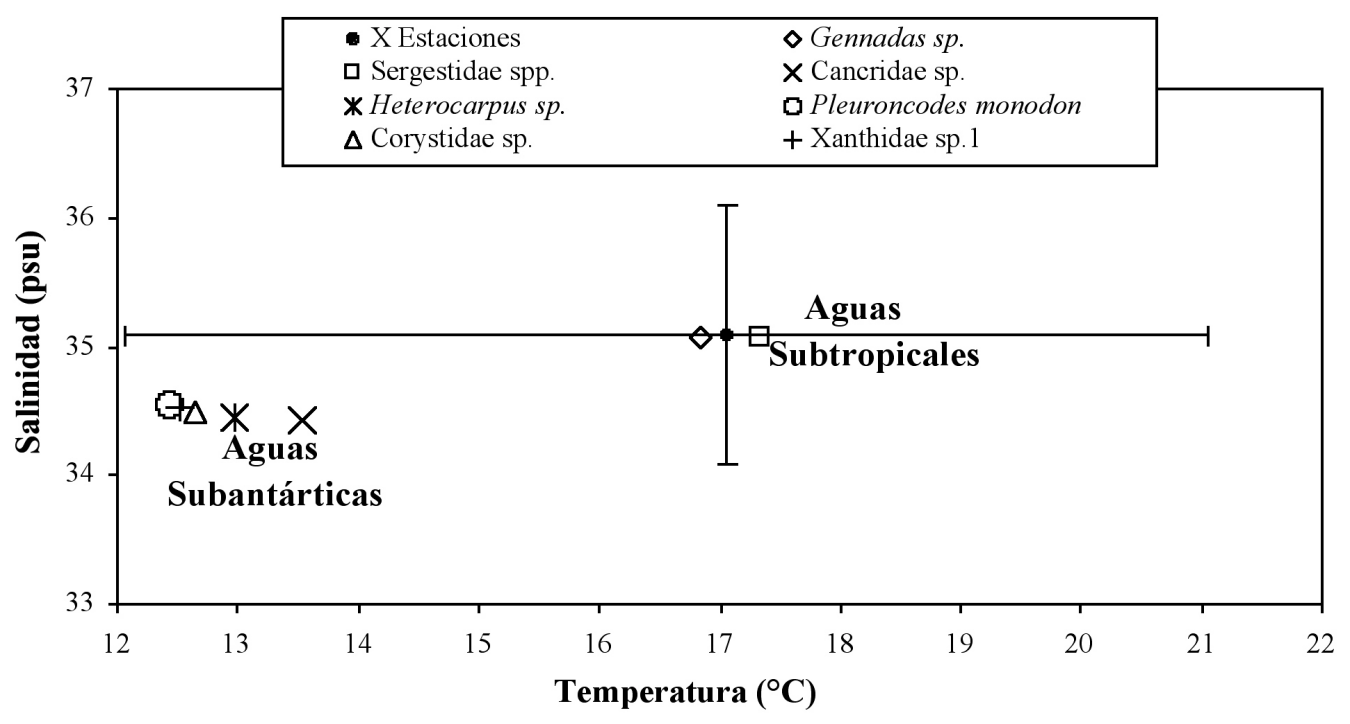

Figura 9. Valor medio y rango de los promedios ponderados verticales $(0-100 \mathrm{~m})$ de temperatura y salinidad en el transecto oceánico entre Caldera-isla de Pascua y distribución termo-halina de las larvas de decápodos más abundantes durante octubre de 1999.

Figure 9. Mean value and range of the temperature and salinity vertical weighted values $(0-100 \mathrm{~m})$ in the oceanic transect between Caldera-Easter Island and termo-haline distribution for the most abundant decapod larvae, during October 1999.

(Scyllaridae) y larvas de carideos de los géneros Oplophorus, Nematocarcinus, Periclimenes y Lysmata, los que cuentan con especies endémicas en los montes submarinos y en sus islas asociadas, como la isla Salas y Gómez e isla de Pascua (Burukovsky,1990; Vereschaka, 1990; Parin et al., 1997; Poupin, 2003).

Así también, se amplió el rango de distribución de las larvas Thalassinidae morfo D.I., forma larval que corresponde a la descrita por Gurney (1938), quien señaló su distribución desde el Atlántico norte hasta la costa este de África, abriendo de esta manera una nueva problemática respecto a las posibles vías de dispersión larval, invasión de especies e influencia entre el Pacífico sudoccidental y sudoriental.

Sin embargo, las variaciones en la riqueza taxonómica de la zona B podrían tener explicación al considerar la ubicación longitudinal de las elevaciones más relevantes dentro de la dorsal de Salas y Gómez (Fig. 10), ubicada entre los $25^{\circ}$ y $26^{\circ} \mathrm{S}$ (Parin et al., 1997), apreciando que en las estaciones cercanas a montes submarinos hubo un aumento en el índice de diversidad, implicando un pequeño incremento en la abundancia relativa de larvas. Esto se relacionaría con las características oceanográficas particulares de estos montes, como son pulsos de surgencia a microescala, que implican fuertes aumentos de la productividad biológica, así como la advección de zooplancton de aguas más profundas hacia aguas superficiales (Vereschaka, 1990; 1995; Parin et al., 1997).

Esto último, concuerda con lo observado en el presente trabajo, donde se constató la presencia de larvas de decápodos de hábitos bentodemersales y bentónicos, pertenecientes a los géneros Nematocarcinus, Periclimenes y Lysmata, que se capturaron en las estaciones 16, 26 y 32 respectivamente, localizadas en la cercanía de montes submarinos.

\section{Asociaciones entre unidades taxonómicas}

Vereschaka $(1990,1995)$ divide en dos grupos o "ensambles" las larvas de decápodos presentes en los montes submarinos de la dorsal Salas y Gómez, considerando a todos los estados planctónicos de especies ampliamente distribuidas en el océano como un grupo y a las especies obligatoriamente conectadas con la cima de los montes submarinos como otro grupo, como es el caso de las larvas de decápodos bentónicos de dichos sectores. Esto coincide con los resultados obtenidos, en que median- 
te el análisis ecológico inverso tipo R (Fig. 5), se conformaron dos grupos principales de larvas de decápodos, equivalentes a los señalados por Vereschaka (1990, 1995).

El primer grupo consideró las larvas de Gennadas sp. y de Sergestidae spp., mostrando un alto grado de asociación entre ellas, coincidiendo con el primer grupo señalado por Vereschaka (1990, 1995), siendo larvas de especies ampliamente distribuidas en los océanos (Omori, 1974; Raymont, 1983; Guzmán, 1999; Guzmán \& Wicksten, 2000). El segundo grupo, incluyó al resto de las unidades taxonómicas encontradas a lo largo del transecto oceánico, que correspondería al grupo de especies asociadas a los montes submarinos.

En el presente trabajo, este último grupo se subdividió en dos, debido a que la sección de estudio consideró tanto el SCH y la plataforma continental, como a las aguas subtropicales y los montes submarinos, por lo que las formas larvales correspondieron a especies de decápodos asociados al bentos de ambos sistemas (Fig. 5). Esto indicaría un alto grado de asociación entre las especies y los grupos de estaciones formados en el análisis Q, quedando fuera sólo las larvas de Gennadas sp. y Sergestidae spp., que fueron las únicas presentes en ambos sistemas, debido a su amplio rango de distribución señalado.

\section{Larvas de decápodos y variables ambientales}

Los resultados obtenidos han permitido correlacionar características físicas de las aguas del Pacífico sudoriental con los patrones de abundancia y distribución de las distintas larvas de decápodos en una amplia zona casi inexplorada. Este tipo de estudios, generalmente, están orientados a describir patrones ambientales en una escala espacial (Coull \& Bell, 1983).

En aguas costeras chilenas, Ulloa \& Palma (1998) relacionaron la temperatura y salinidad con la distribución y abundancias de larvas de decápodos porcelánidos en la bahía de Valparaíso, mostrando relaciones significativas y directas entre éstas. Esta situación se asemeja a la descrita por Escribano e Hidalgo (2000) y Escribano et al. (2002) para los copépodos asociados al SCH en la bahía de Mejillones, quienes describen una fuerte relación entre las mayores abundancias de copépodos y las características propias de las aguas con pulsos de surgencia, como son aguas de menor temperatura y baja concentración de oxígeno disuelto.

Las correlaciones entre la distribución y abundancia relativa de las larvas, con las variables ambientales, mostraron el efecto del incremento de temperatura y en menor grado de la salinidad sobre la abundancia, que disminuyó con el incremento pau-

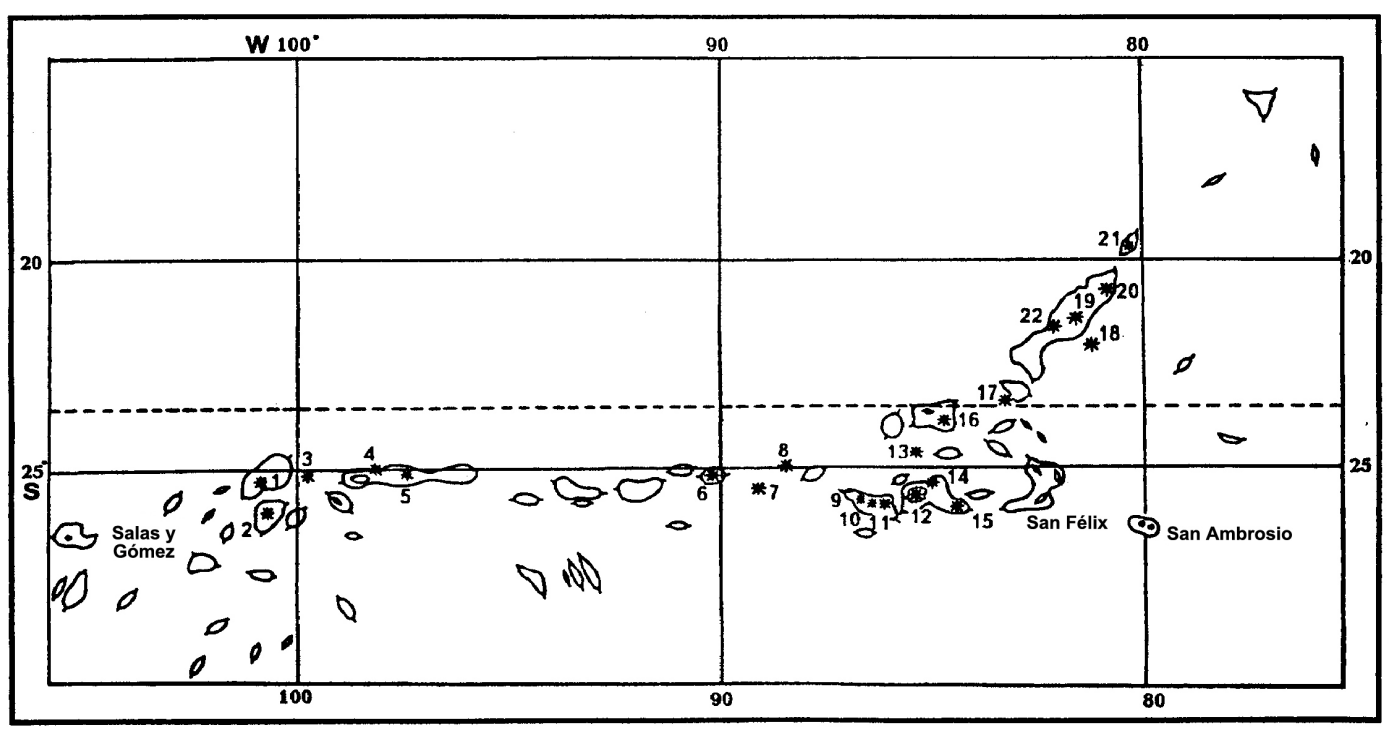

Figura 10. Ubicación de los montes submarinos en la dorsal Salas y Gómez a $\operatorname{los} 25^{\circ}-27^{\circ} \mathrm{S}$, cerca del área de estudio (redibujado de Parin et al., 1997).

Figure 10. Location of the seamounts in the Salas y Gómez ridges between the $25^{\circ}-27^{\circ} \mathrm{S}$, near of sampling area (redraw after Parin et al., 1997). 
latino de los promedios ponderados de estas variables. Siendo esta situación similar a lo reportado por Fernández et al. (2002) para los eufáusidos en bahía de Mejillones, donde la abundancia estuvo inversamente relacionada con los valores de temperatura además del oxígeno disuelto.

Si bien la sumatoria de requerimientos de las diferentes larvas de decápodos es mayor que la magnitud de variabilidad de temperatura y salinidad en este estudio (Anger, 2001), sus abundancias tendrían relación con las variables consideradas, mostrando un mayor grado de determinación por la temperatura y en un grado menor por la salinidad, siendo este patrón observable a niveles de mesoescala (Haury et al., 1978; Mann \& Lazier, 1991). Esto tendría relación con lo descrito por diversos autores, sobre la dispersión y retención de larvas de decápodos en los océanos, las que tienen un gran número de respuestas funcionales a meso y a microescala en el ambiente, lo cual genera diferentes patrones de dispersión determinados por grandes patrones hidrográficos (Cronin \& Forward, 1982; Sulkin, 1984; Forward, 1987, 1990; Hines, 1986; McConaugha, 1992; Escribano et al., 2002).

Anger (2001) ha señalado que la temperatura, salinidad y oxígeno disuelto, entre otros, serían factores ecológicos claves que influyen sobre el desarrollo y otros procesos fisiológicos en las larvas de decápodos, siendo una regla general que el rango de tolerancia de estos factores esté asociado con la distribución geográfica de las especies.

Basándose en esto, los distintos grupos dominantes de larvas, mostraron distintos grados de correlación que reflejaron el rango de tolerancia de las larvas en dirección longitudinal, con respecto a la abundancia relativa de éstas a lo largo del transecto. Así, por ejemplo, las larvas de las familias Xanthidae sp 1. y Cancridae sp. se relacionaron con bajos valores de temperatura y salinidad, siendo estas características relacionadas a un ambiente con pulsos de surgencia costera (Pitts, 1999; Santander et al., 2001; Escribano et al., 2002).

La abundancia de larvas de Sergestidae no se relacionó significativamente con las variables consideradas, lo que podría tener relación con la amplia distribución de estos organismos en aguas del Pacífico sudoriental (Omori, 1974). Es probable que otras variables puedan estar relacionadas con la abundancia de este grupo, siendo interesante destacar la ausencia de estas larvas en las estaciones más cercanas al continente (Est. 1 y 2), con bajos valo- res de temperatura y salinidad.

En síntesis, tales condiciones estarían definiendo la distribución longitudinal de los grupos de larvas de decápodos a mesoescala, siendo la temperatura la que se relacionó con el $71 \%$ de los taxa dominantes, afectando la abundancia y distribución longitudinal de las larvas de decápodos continentales, como fueron Cancridae sp., Xanthidae sp. 1, Heterocapus sp. y Pleuroncodes monodon, asociados a aguas frías subantárticas del SCH. Por otra parte, las larvas de Sergestidae y Gennadas sp., se asociaron a aguas cálidas subtropicales (Fig. 9), siendo coincidentes con lo señalado por Anger (2001), observando una respuesta estenotermo-halina en las larvas de decápodos asociados a aguas frías y una respuesta euritermo-halina en aquellas que viven en aguas templadas, destacando que cada propiedad física y biológica puede variar dentro de un estrecho rango espacio-temporal (Haury et al., 1978).

\section{AGRADECIMIENTOS}

Los autores expresan sus sinceros agradecimientos al Comité Oceanográfico Nacional (CONA) por el desarrollo de los cruceros de investigación científica marina Cimar 5 y 6, Islas Oceánicas, los cuales han permitido la elaboración de trabajos que contribuyen al conocimiento de ecosistemas marinos oceánicos. De igual manera, a los profesores de la Universidad Arturo Prat, Edgardo Santander y Rosalino Fuenzalida, por su colaboración y consejos, a los profesores Walter Sielfeld y Raúl Soto por su apoyo a las nuevas generaciones de científicos, y a mi profesor y amigo Guillermo Guzmán por sus críticos comentarios y literatura facilitada para elaborar este trabajo. Finalmente, agradecemos los comentarios de los tres revisores anónimos.

\section{REFERENCIAS}

Albornoz, L. \& I. Wehrtmann. 1997. Descripción y clave de los primeros estadios larvales de camarones (Decapoda: Hippolytidae; Alpheidae, Rhynchocinetidae) de aguas costeras de Chile. Invest. Mar., Valparaíso, 25: 121-133.

Anger, K. 2001. The biology of decapod crustacean larvae. R. Vonk (ed.). Crustacean Issues 14. A. Balkema, Amsterdam, 419 pp. 
Arana, P. 1987. Perspectivas históricas y proyecciones de la actividad pesquera realizada en el archipiélago de Juan Fernández, Chile. En: J.C. Castilla (ed.). Islas oceánicas chilenas: conocimiento científico y necesidades de investigaciones. Ediciones Universidad Católica de Chile, Santiago, pp. 319353.

Aste, A. \& M. A. Retamal. 1984. Desarrollo larval de Neotrypaea uncinata H. Milne Edwards, 1837 (Decapoda, Callianasidae) bajo condiciones de laboratorio. Gayana, Zool., 48(1-2): 41-56.

Avaria, S., S. Palma, H. Sievers \& N. Silva. 1989. Revisión sobre aspectos oceanográficos físicos, químicos y planctónicos de la bahía de Valparaíso y áreas adyacentes. Biol. Pesq., 18: 67-96.

Báez, P. 1973. Larvas phyllosoma del Pacífico sur oriental (Crustacea, Macrura, Scyllaridea). Rev. Biol. Mar., 15(1): 115-130.

Báez, P. 1997. Key to the families of decapod crustacean larvae collected off northern Chile during an El Niño event. Invest. Mar., Valparaíso, 25: $167-179$.

Báez, P. \& J. Martin. 1992. Decapod crustacean larvae collected off northern Chile during el Niño event (February-March, 1983). Contr. Sci., Nat. Hist. Mus. Los Angeles County, 432: 1-10.

Boschi, E. 1981. Larvas de Crustacea Decapoda. En: D. Boltovskoy (ed.). Atlas de zooplancton Atlántico Sudoccidental y métodos de trabajo con el zooplancton marino. Inst. Nac. Invest. Des. Pesq. (INIDEP), Mar del Plata, pp. 699-718.

Brower, J., J. Zar \& C. Von Ende. 1997. Field and laboratory methods for general ecology. McGrawHill, Boston, 273 pp.

Burukovsky, R. 1990. Shrimps from the Sala y Gomez and Nazca Ridges. En: A.N. Mironov \& J.A. Rudjacov (eds.). Plankton and benthos from the Nazca and Sala-y-Gomez submarine ridges. Trud. Inst. Okeanol. Acad. Sci., Moscow, 124: 187-217.

Calazans, D. 1993. Key to the larvae and decapodids of genera of the infraorder Penaeidea from the Southern Brazilian coast. Nauplius, 1: 45- 62.

Coull, B. \& S. Bell. 1983. Biotic assemblages: populations and communities. En: D. E. Bliss (ed.). The Biology of Crustacea, vol 7. Academic Press, New York, pp. 283-319.
Crain, J. \& P. McLaughlin. 1993. Larval, postlarval and early juvenile development in Pagurus venturensis Coffin, 1957 (Decapoda: Anomura: Paguridae) reared in the laboratory, with a redescription of the adult. Bull. Mar Sci., 53(3): 985-1012.

Criales, M. \& M. McGowan. 1993. Occurrence and description of mysis larvae of a mesopelagic shrimp species of the genus Gennadas (Crustacea: Decapoda: Aristeidae) from coastal water of South Florida. Bull. Mar. Sci., 53(3): 973-984.

Criales, M. \& M. McGowan. 1994. Horizontal and vertical distribution of Penaeidean and Caridean larvae and micronektonic shrimps in the Florida keys. Bull. Mar Sci., 54(3): 843-856.

Cronin, T. \& R. Forward. 1982. Tidally timed behavior: effects of larval distributions in estuaries. En: V. Kennedy (ed.). Estuarine comparisons. Academic Press, New York, pp. 505-520.

Escribano, R. \& P. Hidalgo. 2000. Spatial distribution of copepod in the north of the Humboldt Current region off Chile during coastal upwelling. J. Mar. Biol. Ass. UK., 80: 283-290.

Escribano, R., V. Marín, P. Hidalgo \& G. Olivares. 2002. Physical-biological interactions in the pelagic ecosystem of the nearshore zona of the northern Humboldt current system. En: J.C. Castilla \& J. Largier (eds.). The oceanography and ecology of the nearshore and bays in Chile. Ediciones Universidad Católica de Chile, Santiago, pp. 145-175.

Fagetti, E. 1960. Primer estadio larval de cuatro crustáceos braquiuros de la bahía de Valparaíso. Rev. Biol. Mar., 10: 143-154.

Fagetti, E. \& I. Campodonico. 1971. Larval development of the red crab Pleuroncodes monodon (Decapoda, Anomura, Galatheidae) under laboratory conditions. Mar. Biol., 8(1): 70-81.

Felder, D., J. Martin \& J. Goy. 1985. Patterns in early postlarval development of decapods. En: A.M. Wenner (ed.). Larval growth. Crustacean Issues 2, A. Balkema, Boston, pp. 163-226.

Fernández, D., R. Escribano P. Hidalgo. 2002. Distribución de eufáusidos en el sistema de surgencia frente a la península de Mejillones $\left(23^{\circ} \mathrm{S}\right)$ asociada a condiciones previas y durante El Niño 1997 98. Invest. Mar., Valparaíso, 30(1): 25-43. 
Field, J., K. Clarke \& M. Warwick. 1982. A practical strategy for multispecies distribution patterns. Mar. Ecol. Prog. Ser., 8: 17-52.

Forward, R. 1987. Larval release rhythms of decapod crustaceans: an overview. Bull. Mar. Sci., 41: 165176.

Forward, R. 1990. Behavioral responses of crustacean larvae to rates of temperatures change. Biol. Bull., 178: 195-204.

Frontier, S. 1981. Tratamiento de los datos. En: D. Boltovskoy (ed.). Atlas de zooplancton Atlántico Sudoccidental y métodos de trabajo con el zooplancton marino. Inst. Nac. Invest. Des. Pesq. (INIDEP), Mar del Plata, pp. 169-188.

Gore, R. 1979. Larval development of Galathea rostrata under laboratory conditions, with a discussion of larval development in the Galatheidae (Crustacea Anomura). US Fish. Bull., 76(4): 781806.

Griffiths, F. \& S. Brandt. 1983. Mesopelagic Crustacea in and around a warm-core eddy in the Tasman Sea off eastern Australia. Aust. J. Mar. Freshwat. Res., 34: 609-623.

Gurney, R. 1938. Larvae of decapod Crustacea. Part V Nephropidae and Thalassinidea. Discovery Rep., 17: 291-344.

Gurney, R. \& M. Lebour. 1940. Larvae of Decapod Crustacea. Part VI. The Genus Sergestes. Discovery Rep., 20:1-68.

Guzmán, G. 1999. Nuevos registros de camarones sergéstidos (Crustacea; Decapoda; Dendrobranchiata) en el norte de Chile. Invest. Mar., Valparaíso, 27: 87-91.

Guzmán, G. \& M. Wicksten. 2000. The subfamily Benthesicyminae Bouvier, 1908 (Decapoda, Dendrobranchiata) in northern Chile $\left(18^{\circ}\right.$ to $\left.22^{\circ} \mathrm{S}\right)$. Crustaceana, 73(8): 925-931.

Hart, J. 1971. Key to planktonic larvae of families of decapod Crustacea of British Columbia. Syesis, 4: 227-234.

Haury, L., J. McGowan \& P. Wiebe. 1978. Patterns and processes in the time-space scales of plankton distributions. En: J.H. Steele (ed.). Spatial pattern in plankton communities. Ser. IV. Plenum Press, New York, 3: 277-327.
Heldt, H. 1938. La réproduction chez les crustacés décapodes de la famille des penéidés. Ann. Inst. Oceanogr., 18(2): 31-306.

Hendrickx, M. \& F. Estrada-Navarrete. 1996. Los camarones pelágicos (Crustacea: Dendrobranchiata y Caridea) del Pacífico Mexicano. CONABIO, Inst. Cs. Mar Limnol., UNAM, México, 157 pp.

Hines, A. 1986. Larval patterns in the life histories of brachyuran crabs (Crustacea, Decapoda, Brachyura). Bull. Mar. Sci., 39: 444-466.

Hiyodo C., A. Fransozo \& M. Negreiros-Franzoso. 1994. Larval development of the spider crab Acanthonyx petiverii $\mathrm{H}$. Milne Edwards, 1834 (Decapoda, Majidae) in the Laboratory. Crustaceana, 66(1): 53-66.

Hong, S. \& R. Ingle. 1987. Larval development of the circular crab, Atelecyclus rotundatus (Olivi) (Crustacea: Brachyura: Atelecyclidae) reared in the laboratory. J. Nat. Hist., 21: 1539-1560.

Iwasaki, N. \& T. Nemoto. 1987. Pelagic shrimp (Crustacea: Decapoda) from the Southern Ocean between $150^{\circ} \mathrm{E}$ and $117^{\circ} \mathrm{E}$. Mem. Natl. Inst. Polar Res. Ser E., 38: 1-40.

Ito, M. \& J. Lucas. 1990. The complete larval development of the scyllarid lobster, Scyllarus demani Holthuis, 1946 (Decapoda, Scyllaridae), in the laboratory. Crustaceana, 58(2): 144-167.

Johnson, M. \& W. Lewis. 1942. Pelagic larval stages of the sand crabs Emerita analoga (Stimpson), Blepharipoda occidentalis (Randall) and Lepidopa myops (Stimpson). Biol. Bull., 83: 67-87.

Knight, M. \& M. Omori. 1982. The larval development of Sergestes similis Hansen (Crustacea, Decapoda, Sergestidae) reared in the laboratory. US Fish. Bull., 8(2): 217-243.

Martin, J. \& G. Davis. 2001. An updated classification of the recent Crustacea. Nat. Hist. Mus., Los Angeles County, Science series, 39: 132 pp.

Makarov, R. 1968. On the larval development of the genus Sclerocrangon G.O. Sars (Caridea, Crangonidae). Crustaceana, Suppl. 2: 27-37.

Mann, K., \& J. Lazier. 1991. Dynamics of marine ecosystems. Biological-physical interactions in the oceans. Blackwell Scientific Publications, Boston, $466 \mathrm{pp}$. 
McConaugha, J. 1992. Decapod larvae: dispersal, mortality, and ecology. A working hypothesis. Amer. Zool., 32: 512-523.

McGowan, J. 1971. Oceanic biogeography of the Pacific. En: B.M. Funnell \& W.R. Riedel (eds.). The micropaleontology of oceans. Cambridge University Press, London, pp. 3-74.

McWilliam, P. \& B. Phillips. 1983. Study of phyllosoma larvae and other crustacean macrozooplankton associated with eddy J, a warmcore eddy off south-eastern Australia. Aust. J. Mar. Freshwat. Res., 34: 653- 663.

Menon, P. 1972. Decapod Crustacea from the International Indian Ocean Expeditions: The larval development of Heterocarpus (Caridea). J. Zool., 167: 371-397.

Moraga, J. \& J. Olivares. 1996. Hidrografía del área costera de isla de Pascua, Mayo 1994. Cienc. Tecnol. Mar, 19: 47-56.

Mujica, A. 1993. Zooplancton de las aguas circundantes a la isla de Pascua (2708' S-109²6'W). Cienc. Tecnol. Mar, 16: 55-61.

Mujica, A. \& M. Medina. 1997. Larvas de crustáceos decápodos de los canales australes de Chile (41³0’-46³0’S). Cienc. Tecnol. Mar, 20: 147-154.

Natunewicz, C. \& C. Epifanio. 2001. Spatial and temporal scales of patches of crab larvae in coastal waters. Mar. Ecol. Prog. Ser., 212: 217-222.

Olivares, J. \& J. Moraga. 1993. Contribución a la descripción de las condiciones oceanográficas del área costera de isla de Pascua. Cienc. Tecnol. Mar, 16: 47- 54.

Omori, M. 1974. The biology of pelagic shrimps in the oceans. Adv. Mar. Biol., 12: 233-324.

Palma, S. 1976. Meroplacton de la región de Valparaíso. Cienc. Tecnol. Mar, 2: 99-116.

Palma, S. 1980. Larvas de crustáceos decápodos capturadas frente a la costa de Valparaíso. Invest. Mar., Valparaíso, 8(1-2): 129-144.

Palma, S. 1985. Plancton marino de las aguas circundantes al archipiélago de Juan Fernández. En: P. Arana (ed.). Investigaciones marinas en el archipiélago de Juan Fernández. Escuela de Ciencias del Mar, Valparaíso, pp. 59-69.
Palma, S. 1994. Distribución y abundancia de larvas de langostino colorado Pleuroncodes monodon frente a la costa de Concepción, Chile. Invest. Mar., Valparaíso, 22: 13-29.

Palma, S., J. Meruane \& A. Mujica. 1976. Observaciones sobre el meroplancton del archipiélago de Juan Fernández. Enero 1974. Cienc. Tecnol. Mar, 2: $117-126$.

Parker, H. 1985. A guide to the larvae of New Zealand shallow water Caridea (Crustacea, Decapoda, Natantia). Zool. Publ. Victoria University of Wellington, 78: 1-20.

Parin, N., A. Mironov \& K. Nesis. 1997. Biology of Nazca and Sala y Gómez submarine ridges, an outpost of the Indo-west Pacific fauna in the eastern Pacific ocean: composition and distribution of the fauna, its communities and history. Adv. Mar. Biol., 32: $147-230$.

Parson, T., M. Takahashi \& B. Hargrave. 1984. Biological oceanographic processes. Pergamon Press, New York, 330 pp.

Paula, J. 1996. A key and bibliography for the identificacion of zoeal stages of brachyuran crabs (Crustacea, Decapoda, Brachyura) from the Atlantic coast of Europe. J. Plankton Res., 18(1): 17-27.

Pitts, P. 1999. Effects of summer upwelling on the abundance and vertical distribution of fish and crustacean larvae off central Florida's Atlantic coast. J. Exp. Mar. Biol. Ecol., 235: 135-146.

Poupin, J. 2003. Crustacea Decapoda and Stomatopoda of Easter Island and surrounding areas. A documented checklist with historical overview and biogeographic comments. Atoll Res. Bull., 500: 1-56.

Raymont, J. 1983. Plankton and productivity in the oceans. Zooplankton. Pergamon Press, Oxford, 824 pp.

Rivera, J. \& G. Guzmán. 2002. Descripción de larvas mysis de tres especies de camarones mesopelágicos del género Gennadas (Decapoda: Aristeidae) en las aguas del Pacífico sudoriental. Invest. Mar., Valparaíso, 30(2): 33-44.

Rosales, S. 1992. Estructura comunitaria horizontal de copépodos calanoídeos y características hidrográficas entre Los Vilos y Valparaíso, duran- 
te enero de 1990. Tesis de Oceanografía, Escuela de Ciencias del Mar, Universidad Católica de Valparaíso, $85 \mathrm{pp}$.

Santander, E., L. Herrera \& J. Pizarro. 2001. Fluctuación diaria del fitoplancton en la capa superficial del océano durante la primavera de 1997 en el norte de Chile (20¹8'S): I. Biomasa pigmentaria. Rev. Biol. Mar., 36(2): 141-153.

Sastry, N. 1983. Pelagic larval ecology and development. En: D.E. Bliss (ed.). The biology of Crustacea, Vol 7. Academic Press, New York, pp. 213-282.

Seridji, R. 1995. On a collection of decapod larvae from the gulf of Aqaba off the Jordian coast. I. Thalassinidea and Anomura. Crustaceana, 68: 281220.

Sievers, H. \& N. Silva. 1975. Masas de agua y circulación en el océano Pacífico sudoriental, latitudes $18^{\circ} \mathrm{S}-33^{\circ} \mathrm{S}$. (Operación Oceanográfica "MarChile VIII”). Cienc. Tecnol. Mar, 1: 7-67.

Silva, N. 1985. Algunas características físicas y químicas de las masas de agua alrededor de las islas Robinson Crusoe y Santa Clara. Invest. Mar., Valparaíso, 6(4): 49-72.

Silva, N. 1992. Condiciones oceanográficas alrededor de la isla de Pascua durante la primavera de 1979. Cienc. Tecnol. Mar, 15: 21-30.

Silva, N. \& D. Konow. 1975. Contribución al conocimiento de las masas de agua en el Pacífico sudoriental, Expedición Krill. Crucero 3-4 julioagosto 1974. Rev. Com. Perm. Pacífico Sur, 3: 6375.

Silva, N. \& H. Sievers. 1974. Masas de agua, velocidad geostrófica y transporte de volumen entre Valparaíso e isla Robinson Crusoe (Océano Pacífico Sudoriental). Rev. Com. Perm. Pacífico Sur, 2: 102120

Recibido: 22 octubre 2003; Aceptado: 27 julio 2004
Strub, T., J. Mesías, V. Montecino, J. Rutllant \& S. Salinas. 1998. Coastal ocean circulation off western south America. Coastal segment (6 E). The Sea, 11: 273-313.

Sulkin, S. 1984. Behavioral basis of depth regulation in the larvae of brachyuran crabs. Mar. Ecol. Prog. Ser., 15: 181-205.

Ulloa, R. \& S. Palma. 1998. Distribución larval de Petrolisthes violaceus, $P$. laevigatus y Allopetrolisthes angulosus en el plancton de la bahía de Valparaíso (Crustacea, Anomura, Porcellanidae). Rev. Biol. Mar., 33(1): 125-138.

Vereshchaka, A. 1990. Pelagic decapods from semount of Nazca and Sala-y-Gomez ridges. En: A.N. Mironov \& J.A. Rudjacov (eds.). Plankton and benthos from the Nazca and Sala-y-Gomez submarine ridges. Trud. Inst. Okeanol., Acad. Sci., Moscow, 124: 129-155.

Vereschaka, A. 1995. Macroplankton in the nearbottom layer of continental slopes and seamounts. Deep-Sea Res., 42(9): 1639-1668.

Wehrtmann, I. \& P. Báez. 1997. Larvas y estadíos tempranos de desarrollo de crustáceos decápodos de Chile. Descripciones publicadas. Invest. Mar., Valparaíso, 25: 263-276.

Williamson. D. \& K.G. Von Levetsow. 1966. Larvae of Parapagurus diogenes (Whitelegge) and some related species (Decapoda, Anomura). Crustaceana, 14(2): 179-192.

Yeung, C. \& M. McGowan. 1991. Differences in inshore-offshore and vertical distribution of phyllosoma larvae of Panulirus, Scyllarus and Scyllarides in Florida keys in May-June, 1989. Bull. Mar. Sci., 49(3): 699-714.

Zar, J. 1984. Biostatistical analysis. Prentice Hall, New Jersey, 718 pp. 